\title{
The General Growth Logistics of Cell Populations
}

\author{
H. G. Kilian · D. Bartkowiak · D. Kaufmann • \\ R. Kemkemer
}

Published online: 21 May 2008

(C) Humana Press 2008

\begin{abstract}
An increment model based on thermodynamics lays bare that the cell size distributions of archaea, prokaryotes and eukaryotes are optimized and belong to the same universal class. Yet, when a cell absorbs mass or signals are processed, these conditions are disturbed. Relaxation re-installs ideal growth conditions via an exponential process with a rate that slows down with the cell size. In a growing ensemble, a distribution of relaxation modes comes in existence, exactly defined by the universal cell size distribution. The discovery of nanomechanic acoustic activities in cells led us to assume that in a growing ensemble acoustic signals may contribute significantly to the transmission of essential information about growth-induced disturbances to all cells, initiating that way coordinated relaxation. The frequency increases with the cell number shortening the period between successive signals. The completion of rearrangements occurring at a constant rate is thus progressively impaired,
\end{abstract}

H. G. Kilian ( $₫)$

Abteilung Experimentelle Physik, Universität Ulm,

Albert-Einstein Allee 11, 89069 Ulm, Germany

e-mail: hanns-georg.kilian@uni-ulm.de

D. Bartkowiak

Abteilung Strahlentherapie, Universitätsklinik,

Robert-Koch-Straße 6, 89081 Ulm, Germany

e-mail: detlef.bartkowiak@uniklinik-ulm.de

D. Kaufmann

Institut für Humangenetik, Universitätsklinik,

Albert-Einstein Allee 11, 89081 Ulm, Germany

e-mail: dieter.h.kaufmann@uni-ulm.de

R. Kemkemer

Max-Planck-Institut für Metallforschung, Heisenbergstr. 3,

70569 Stuttgart, Germany

e-mail: ralf.kemkemer@mf.mgp.de until cellular growth stops, totally. Due to this phenomenon, the so-called "relaxation-frequency-dispersion" cell colonies should exhibit a maximum cell number. In populations with large cell numbers, subsystems, behaving similar-like colonies, should form network-like patterns. Based on these ideas, we formulate equations that describe the growth curves of all cell types, verifying that way the general nature of the growth logistics.

Keywords Cell multiplication · Colonies · Populations · Structure and dynamics - Relaxation · Growth logistics

\section{Introduction}

The growth of multi-cellular living systems is linked via cell division to the growth of individual cells [1]. A phenomenological description of these processes should provide information about cooperation within cell ensembles. If synchronized sequences of cell growth and division occur in an unlimited manner, an exponential relation describes the number of cells and in a semi-log plot a straight-line results, not showing the sigmoidal bending of experimental patterns. Various treatments [2-5] present different approaches to a deeper understanding of the observations by modelling universality and scaling. Yet, limits of the characterization are also made out like the question whether and how the cell ensemble structure exercises an influence on the growth process.

Earlier treatments [6-8] identify the cell size distributions deduced by Kilian et al. [8] and Schlote [9] as optimized patterns. Moreover, expressed in dimensionless variables all of them coincide with one another. In order to understand this striking symmetry, a quantitative description of growth is required which accounts for the essential 
features of the structural development. Of course, at the present state of knowledge, this treatment needs sensible simplifications and approximations.

One central assumption is that throughout all stages of their lifecycle, cells tend to keep optimal process conditions, which are disturbed, however, each time when mass is taken up or signals are processed. To re-establish the optimal state, intra-cellular relaxation has to occur. Since the relaxation time increases with the cell size, a relaxation-time-distribution results, determined by the size distribution of the cell ensemble. In addition, whenever a cell grows, the momentary size distribution deviates from its ideal version $[6-8,10]$. All cells have to improve the situation, by collective relaxation.

During these events, communication should coordinate the numerous bio-molecular processes. Intercellularsignalling may occur via cell-to-cell coupling that supports synchrony across the whole population [11]. "Quorumsensing" is the ability of cells to detect and respond to their population density. In any case, growth control needs collective signals that exhibit at least these features: (a) Signals should inform all cells of a growing ensemble about the actual situation. (b) They should thus be transmitted fast enough in respect to the mean rate of molecular reaction and reorganization. (c) Via absorption and emission, cells should be able to take individual action. (d) A frequency spectrum should allow to generate precise messages.

In order to develop under these conditions a phenomenological model, we refer to the observations of nano-mechanical motions of the cell wall of Saccharomycescerevisiae [12]. Systematic studies lead to the conclusion that this "acoustic breathing" is a metabolically driven process. One might then assume that cellular absorption of biomass causes acoustic signals. Yet, the concerted activities of all the communicating cells in a colony, including complex effects of intercellular contacts [13], are not known to the last details. We must content ourselves with approximating the succession of signals as an oscillating stress-field with frequencies in the acoustic regime.

During growth, these frequencies increase steadily, thus shortening the period between successive signals. Molecular rearrangements that show a constant rate become more and more incomplete. Finally, any further growth is blocked. We call this whole phenomenon "relaxation-frequency-dispersion". In colonies, the number of cells should in principle be restricted to a maximum value. Any population comprised of a number of cells beyond this limit should form a transient network of subsystems with properties resembling those of a single colony.

Based on the above ideas, equations are formulated that allow us to describe the growth of populations for different cell types and under any culture condition, that way revealing notable symmetries.

\section{Stationary Properties}

The Saturation Conditions

Ensembles of cultured cells from all biological kingdoms exhibit cell size distributions with conspicuously similar properties $[9,14]$. By introducing an "increment model", it can be discussed how they may come about [6-8]. We suppose that in a growing cell energetically equivalent molecule clusters are absorbed and relatively fast adjusted to optimal conditions. These "equilibrated clusters" are regarded as particles, dubbed increments of biomass (ICs). Thermodynamics legitimates the assumption that the nutrient solution should contain such increments as preformed particles, that way formulating a virtual state of reference. This characterization obviates the necessity of dealing with the formation of ICs via a not fully known intracellular biochemistry and processes of self-organization.

In terms of this model, growth of a cell is a sequential addition of ICs in a re-iterated uniform reaction. The reaction-energy $\Delta u_{\mathrm{c}}$ of an IC as difference between the energy $u_{\mathrm{c}}$ in the cell and the energy $u_{\mathrm{n}}$ in the nutrient is $\Delta u_{\mathrm{c}}=u_{\mathrm{c}}-u_{\mathrm{n}}$.

There are many reasons to suspect that keeping optimal growth conditions needs unceasing re-adjustments. They seem to happen analogous to the adaptation of equilibrium in a streaming gas. Within every volume element, relaxation installs thermal equilibrium, independent of the stationary collective movement of all particles. In cells, disturbance of the optimal internal structure occurs when biomass is absorbed. Virtually independent of the quasi-stationary collective growth of the total ensemble, relaxation should always arrange optimal process conditions.

The disturbances are likely to be small enough to justify the application of irreversible thermodynamics. At constant absolute temperature $T$ and pressure $p$, the differential of the Gibbs-free-energy $\left(d \mathrm{~g}_{y}\right)_{T, p}$ within a growing cell comprised of $y_{a}$ ICs with $y=y_{a}-1$ contacts, can according to [15] be defined by

$\left(d \mathrm{~g}_{y}\right)_{T, p}=-A_{y} d \xi_{y}(t)$

$\xi_{y}(t)$ is the reaction coordinate. The affinity $A_{y}>0$ measures the distance from stationary conditions at $A_{y}=0$. If, on average, each increment experiences the same growthinduced disturbance, the affinity can be written as $A_{y}=y A_{0}$. The affinity per increment $A_{0}$ is then equal to

$A_{0}=-\sum v_{k} \mu_{k}=\mu_{n}-\mu_{c} ; \quad v_{n}=-1 ; \quad v_{c}=1$.

The chemical potential of an IC in the nutrient solution is $\mu_{\mathrm{n}}$, and in the cell $\mu_{\mathrm{c}} . v_{j}$ are the stoichiometric coefficients: the negative term $\left(v_{\mathrm{n}}=-1\right)$ indicates that an IC disappears from the reservoir when it is linked in a cell 
$\left(v_{\mathrm{c}}=+1\right)$. With the definition $\mu_{j}=u_{j}-T s_{j}(j=\mathrm{c}, \mathrm{n})$ the relations per IC emerge

$$
\begin{array}{r}
u_{\mathrm{c}}-u_{\mathrm{n}}+A_{0}=T\left(s_{\mathrm{c}}-s_{\mathrm{n}}\right), \\
\Delta u_{o}=\Delta u_{\mathrm{c}}+A_{0}=T \Delta s_{\mathrm{c}} .
\end{array}
$$

Since in every cell the values of the reaction energy $\Delta u_{\mathrm{c}}$ und the affinity $A_{0}$ are supposed to be invariant, the reaction energy $\Delta u_{y}$ of a cell with $y$ contacts is equal to $\Delta u_{y}=y \Delta u_{0}$. The reaction entropy $\Delta s_{y}=s_{y}-s_{y n}$ is the difference of the entropy $s_{y}$ of $y_{a}$ ICs in the cell with $y$ contacts and the entropy $s_{y n}$ of these ICs in the reservoir. Postponing the explicit formulation of $\Delta s_{y}$, the "saturation conditions" emerge

$\Delta u_{y}=y \Delta u_{0}=T \Delta s_{y} ; \quad y_{\min } \leq y \leq y_{\max }$.

The smallest cells have $y_{\min }$ contacts; $y_{\max }$ counts the contacts in cells of maximum size. Under optimal process conditions, the entropy term $T \Delta s_{y}$ and the reaction energy $\Delta u_{y}$ should become equal for each cell size $\left(y_{\min } \leq y\right.$ $\left.\leq y_{\max }\right)$. Via ever-present structural fluctuations, the entropy should rapidly adapt to the value demanded according to Eq. 4.

\section{Cell Size Distributions}

Under these circumstances, cell ensembles should display topologically identical cell size distributions. To verify this statement, the total reaction entropy $\Delta s_{y}$ must be given in greater detail. Assumed to be comprised of the intracellular contribution $\Delta s_{y i}$ and the mixing entropy $\Delta s_{y \text { mix }}$

$\Delta s_{y}=\Delta s_{y i}+\Delta s_{y \operatorname{mix}}$,

we have to explain these components.

As approximation, independent structural fluctuations in three orthogonal directions within a cell are supposed to determine $\Delta s_{y i}$. The number of microstates $\Omega_{y i}$ in the direction $i$ should then be related to the number of increments $y_{a}=y+1$ according to

$\Omega_{y i}=y_{a}^{\alpha_{i}}=(y+1)^{\alpha_{i}} \approx y^{\alpha_{i}} ; \quad i=1,2,3$.

Empirically, $\alpha_{i}$ accounts for the way how a stationary number of microstates in the direction $i$ is produced. The total number of microstates $\Omega_{y}$ in a cell is then

$\Omega_{y} \cong \Omega_{y 1} \Omega_{y 2} \Omega_{y 3}=y^{\alpha_{1}+\alpha_{2}+\alpha_{3}}=y^{p} ; \quad p=\alpha_{1}+\alpha_{2}+\alpha_{3}$.

Related to the state of reference at $y=1$, the intracellular reaction entropy $\Delta s_{y i}$ is defined by

$\Delta s_{y i}=k_{\mathrm{B}}\left[\ln \left(\Omega_{y}\right)-\ln \left(\Omega_{1}\right)\right]=k_{\mathrm{B}} \ln \left(y^{p}\right)$,

where $k_{\mathrm{B}}$ is Boltzmann's constant. Since cell size distributions are experienced to belong without any exception to the $(p=3)$-class, the intracellular entropy $\Delta s_{y i}$ may be represented as if exactly $y$ configurations are independently activated in each direction $i$ so that $\Omega_{y}=y^{3}(p=3)[6,8]$.

$\Delta s_{\text {mix }}$ arises now since according to thermodynamics, a growing cell colony is a multi-component system with cells in different size as its components [16]. When a cell divides, two smaller daughter cells replace the bigger mother cell. Within more or less tightly packed patterns, growth of a cell enforces cooperative rearrangements of the positions of neighbouring cells that are growing too. Not dominated by diffusion, these not strictly synchronized processes accomplish de facto an ideal mixing of cells of different size. The mixing entropy $\Delta s_{\text {mix }}$ [16] is then given by

$\Delta s_{\text {mix }}=-k_{\mathrm{B}} \sum_{y=y_{\min }}^{y_{\max }} n_{y} \ln \left(x_{y}\right) ; \quad x_{y}=\frac{n_{y}}{\Sigma n_{y}}$.

The fraction $x_{y}$ is defined by relating the number of cells with $y$ contacts $n_{y}$ to the total cell number in the ensemble $\Sigma n_{y}$. Since at the state of reference $\left(\partial s_{\text {mix }} / \partial n_{y}\right)_{r}=0$, the mixing entropy contribution of a cell with $y$ contacts $\Delta s_{y \text { mix }}$ is equal to

$\Delta s_{y \text { mix }}=\left(\partial s_{\text {mix }} / \partial n_{y}\right)_{c}-\left(\partial s_{\text {mix }} / \partial n_{y}\right)_{r}=-k_{\mathrm{B}} \ln \left(x_{y}\right)$.

If smallest cells with $y_{\min }$ contacts are in existence, the parameter $y$ should be replaced by $\Delta y=y-y_{\min }$. Equations 6 and 8 inserted into Eq. 4, the fraction $x_{y}$ of cells with $\Delta y$ contacts $x_{y}$ is obtained to be equal to [7]

$x_{y}=\gamma \Delta y^{p} \exp \left\{-\beta \Delta y \Delta u_{0}\right\}=\gamma \Delta y^{p} \exp \left\{-\beta \Delta y\left(\Delta u_{c}+A_{0}\right)\right\}$

with

$\gamma=\left(\sum_{y=y_{\min }}^{y_{\max }} \Delta y^{p} \exp \left\{-\beta \Delta y \Delta u_{0}\right\}\right)^{-1} ; \quad \beta=\left(k_{\mathrm{B}} T\right)^{-1}$.

Given the parameters $p=3, \Delta u_{0}, A_{0}$ and $y_{\min }$, the set of fractions $x_{y}$ describes a cell-size distribution with optimal properties. When Eq. 9 is re-formulated in terms of the dimensionless variable $\eta$ given by relating the reaction energy per cell $\left(y-y_{\min }\right) \Delta u_{0}$ to the thermal energy $k_{\mathrm{B}} T$

$\eta=\left(y-y_{\text {min }}\right) \Delta u_{0} / k_{\mathrm{B}} T=\left(y-y_{\text {min }}\right) \beta \Delta u_{0}$,

the universal function $\Phi(\eta)$ emerges (7)

$\Phi(\eta)=x_{y} / C_{A}=\eta^{p} \exp \{-\eta\} ; \quad C_{A}=\gamma /\left[\beta \Delta u_{0}\right]^{p}$.

Figure 1 demonstrates that the cell size distributions of archaea, prokaroytes and eukaryotes coincide within the limits of accuracy with the universal $(p=3)$-version of $\Phi(\eta)$, i.e. $p$ should not depend on kinetic or genetic factors. Fluctuation of the intracellular structure according to the $(p=3)$-version of Eq. 12 indicates an unexpected symmetry of the intra-cellular dynamics. 


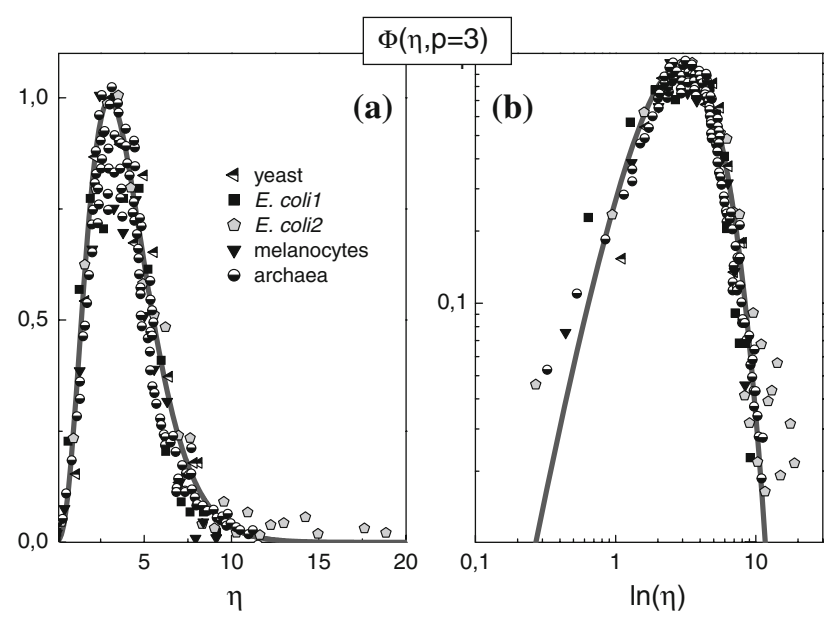

Fig. 1 (a, b) Linear and double logarithmic plot of the $\Phi(\eta, p=3)$ master curves of various cell systems [8]. The solid lines are calculated with the $(p=3)$-version of Eq. 12

\section{The Growth Process}

\section{The Relaxation Mode Factor}

Micoulet et al. [17] deformed single cells and studied relaxation processes. They observed "quasi-Hookian" stress-strain behaviour. When the strain reaches a defined value, the stress shows a first-order exponential decay, characterized by one single relaxation time. The relaxation time should increase with the cell size (see Appendix). In cell ensembles, the cell size distribution should thus cause a relaxation-time-distribution. In order to substantiate this structure-dynamics interrelation, the relaxation time $\tau_{y}$ of a cell with $y$ contacts is written as product of the dimensionless "relaxation mode factor" $\tau_{0 y}$ and the kinetic factor $\tau_{\text {kin }}[8,18]$

$\tau_{y}=\tau_{0 y} \tau_{\text {kin }}$

In the Appendix, these relations are deduced

$\tau_{0 y}=\Omega_{0 y} ; \quad \tau_{\text {kin }}=\frac{1}{a \mu k_{\mathrm{B}} T}$.

The mode factor $\tau_{0 y}$ should be proportional to the number of microstates $\Omega_{0 y}$ within a cell with $y$-contacts that increases with the size in qualitative accordance with Eq. 6 . In contrast, motions of molecular segments should only affect next neighbours, so that $\tau_{\text {kin }}$ should not depend on the ensemble structure. $a$ is Onsager's coefficient. The definition of the parameter $\mu$ is given in the Appendix.

The Relaxation-mode-spectra

Relating the reaction entropy $\Delta s_{y}$ to a state of reference with $\Omega_{n}=1$
$\frac{\Delta s_{y}}{k_{\mathrm{B}}}=\ln \left(\Omega_{0 y}\right)-\ln \left(\Omega_{n}\right)=\ln \left(\Omega_{0 y}\right)$

and combining Eqs. 4, 12 and 14, one arrives at these relations

$\eta=\left(y-y_{\min }\right) \beta \Delta u_{0}=\frac{\Delta s_{y}}{k_{\mathrm{B}}}=\ln \left(\Omega_{0 y}\right)=\ln \left(\tau_{0 y}\right)$.

At constant $p$, the identities emerge

$\Phi(\eta)=\Phi\left(\frac{\Delta s_{y}}{k_{\mathrm{B}}}\right)=\Phi\left(\tau_{0 y}\right)$

with

$$
\begin{array}{r}
\Phi(\eta)=\eta^{p} \exp \{-\eta\}, \\
\Phi\left(\frac{\Delta s_{y}}{k_{\mathrm{B}}}\right)=\left(\frac{\Delta s_{y}}{k_{\mathrm{B}}}\right)^{p} \exp \left\{\frac{-\Delta s_{y}}{k_{\mathrm{B}}}\right\}, \\
\Phi\left(\tau_{0 y}\right)=\frac{\ln ^{p}\left(\tau_{0 y}\right)}{\tau_{0 y}} .
\end{array}
$$

$\Phi(\eta)=\Phi\left(\Delta s_{y} / k_{\mathrm{B}}\right)$ confirms the interrelation between reaction energy and entropy as demanded according to Eq. 4. To fulfil the condition $\Phi(\eta)=\Phi\left(\tau_{0 y}\right)$, the course of the mode spectrum $\Phi\left(\tau_{0 y}\right)$ has to coincide with the universal $(p=3)$-cell size distribution $\Phi(\eta)$, i.e. all cell types should exhibit the same universal $(p=3)$-relaxationmode spectrum $\Phi\left(\tau_{0 y}\right)$.

\section{The Connection Between $\omega_{\mathrm{c}}$ and $t$}

If by absorption of ICs the intra-cellular growth conditions are disturbed with the frequency $\omega_{0}$, the frequency $\omega_{\mathrm{c}}$ of all signals in a colony comprised of $n_{\mathrm{c}}^{\prime}(t)$ cells

$n_{\mathrm{c}}^{\prime}(t)=2^{t / t_{\mathrm{c}}}=\exp \left\{\ln (2)\left(t / t_{\mathrm{c}}\right)\right\}$

is as function of time $t$ related to $t_{\mathrm{c}}$, the period of a cell cycle, set equal to

$\omega_{\mathrm{c}}(t)=\omega_{0} n_{\mathrm{c}}^{\prime}(t)=\omega_{0} 2^{t / t_{\mathrm{c}}}=\omega_{0} \exp \left\{\ln (2)\left(t / t_{\mathrm{c}}\right)\right\}$.

In the time domain, the ideal law of cell multiplication given in Eq. 16 should characterize the exponential part of the growth process. To achieve this, we introduce the parameter $\rho$

$\left(\omega_{\mathrm{c}} / \omega_{0}\right)^{\rho}=\exp \left\{\ln (2)\left(t / t_{\mathrm{c}}\right)\right\}$.

Experimentally, $\rho$ is always observed to be approximately equal to $\ln (2)$ so that we are led to

$\frac{t}{t_{\mathrm{c}}}=\ln \left(\frac{\omega_{\mathrm{c}}}{\omega_{0}}\right)$.

The time dependent functions $n_{\mathrm{c}}^{\prime}(t)$ and $n_{\mathrm{c}}^{\prime \prime}(t)$ are therefore obtained by plotting $n_{\mathrm{c}}^{\prime}\left(\omega_{\mathrm{c}}\right)$ and $n_{\mathrm{c}}^{\prime \prime}\left(\omega_{\mathrm{c}}\right)$ against $t$ given by 
$t=t_{\mathrm{c}} \ln \left(\frac{\omega_{\mathrm{c}}}{\omega_{0}}\right)$

\section{A Principal Limitation}

When a cell absorbs mass, its energy increases, including strain energy that characterizes the initial disturbance via cell deformation; the stress increases spontaneously, evoking acoustic signals which should convey information on the actual local situation. Since in a colony with many cells, nutrient is absorbed in an almost regular sequence [19], these events may be described by a periodic stressfield with the frequency $\omega_{\mathrm{c}}$. If the number of communicating cells grows, $\omega_{\mathrm{c}}$ increases, and the time interval between two successive signals, i.e. the time for molecular reorganization shortens. Since the molecular relaxation processes occur at a constant rate, during growth optimization of the whole ensemble is increasingly constrained. When a network structure develops, reorganization-modes "freeze" selectively starting with the slowest highly cooperative events. The growing reaction energy of the ICs drives then $A_{0}$ to increasing positive values (Eq. 3). According to Fig. 13, this effect is experimentally confirmed. Finally, at the highest frequency $\omega_{\text {cmax }}$, cell growth and division are totally blocked. Usually, a tissue like pattern with an optimal ensemble structure emerges.

\section{An Instructive Analogy}

When a macromolecular network is extended rapidly, the conformational entropy of its flexible chains is reduced because they become stretched. Moreover, they are forced into a non-equilibrium state [20]. The set of optimal conformations has to be installed via relaxation; this requires time since sequential cooperative molecular processes participate. The theory of networks considers the chains as equivalent visco-elastic subsystems. At small strains $\gamma$ the total deformation energy $w^{*}$ per chain is as a function of the elastic strain energy $w^{\prime}$ and the dissipated part $w^{\prime \prime}$ defined by $w^{*}=w^{\prime}+i w^{\prime \prime}=\frac{1}{2} G^{*} \gamma^{2}=\frac{1}{2}\left(G^{\prime}+i G^{\prime \prime}\right) \gamma^{2}$.

In the Hookian regime, the complex modulus $G^{*}$ per chain is then comprised of the real part $G^{\prime}$ and the imaginary part $G^{\prime \prime}$. Since network chains are energyequivalent subsystems, the strain energy $W^{*}$ of the whole network grows proportional to the number of chains

$W^{*}=n w^{*}=n\left(\frac{G^{*}}{2} \gamma^{2}\right)$.

If periodic deformation is applied, the reorganization of the conformation of chains at increasing frequencies is constrained steadily, and finally these processes freeze totally: the network is in the glassy state. Apparently, the phenomenon of relaxation-frequency-dispersion typifies the glass transition of a macromolecular network $[18,21]$.

A growing cell ensemble shows now topologically analogous features since cells operate as equivalent widely autonomous visco-elastic subsystems. They are locally deformed whenever a cell absorbs biomass. Strain-energy is stored, and then in parts dissipated during relaxation. As approximation growing cell ensembles are supposed to be automatically submitted to a collective quasi-periodic deformation, represented by an oscillating stress-field with the increasing frequency $\omega_{\mathrm{c}}$. Optimal process conditions should be adjusted via relaxation, exhibiting analogous to a freezing macromolecular network, the phenomenon of relaxation-frequency-dispersion. We may thus adopt ideas of the theory of rubber elasticity and of the phenomenological classification of relaxation [22].

For the stress $\sigma(t)$ at the amplitude $\sigma_{0}$

$\sigma(t)=\sigma_{0} \exp \left\{i \omega_{\mathrm{c}} t\right\}$

alternating with the frequency $\omega_{\mathrm{c}}$ (Eq. 17), the accompanying strain $\gamma(t)$ is

$\gamma(t)=\gamma_{0} \exp \left\{i \omega_{\mathrm{c}} t-\delta\right\}$.

The phase-shift $\delta$ originates from the dissipation of strain-energy. In a growing cell with $y$ contacts, the real part $G_{y}{ }^{\prime}\left(\omega_{c}\right)$ and the imaginary component $G_{y}{ }^{\prime \prime}\left(\omega_{\mathrm{c}}\right)$ of the complex modulus $G_{y}{ }^{*}\left(\omega_{\mathrm{c}}\right)$ should be defined by [22]

$G_{y}^{\prime}=\Delta G^{\prime} \frac{\omega_{\mathrm{c}}^{2} \tau_{y}^{2}}{1+\omega_{\mathrm{c}}^{2} \tau_{y}^{2}} ; \quad G_{y}^{\prime \prime}=\Delta G^{\prime} \frac{\omega_{\mathrm{c}} \tau_{y}}{1+\omega_{c}^{2} \tau_{y}^{2}}$.

$\Delta G^{\prime}=G^{\prime}{ }_{\max }-G_{\text {min }}^{\prime}$ is the "relaxation-strength", as the difference between the maximum value of the modulus at the largest frequency and its minimum under static conditions. In order to describe the growth of a cell ensemble, it is appropriate to introduce the complex function $X_{y}^{*}\left(\omega_{\mathrm{c}} \tau_{\mathrm{y}}\right)$ per cell

$$
\begin{gathered}
X_{y}^{*}\left(\omega_{\mathrm{c}} \tau_{y}\right)=X_{y}^{\prime}\left(\omega_{\mathrm{c}} \tau_{y}\right)+i X_{y}^{\prime \prime}\left(\omega_{\mathrm{c}} \tau_{y}\right) \\
X_{y}^{\prime}\left(\omega_{\mathrm{c}} \tau_{y}\right)=\frac{G_{y}^{\prime}}{\Delta G^{\prime}}=\frac{\omega_{\mathrm{c}}^{2} \tau_{y}^{2}}{1+\omega_{\mathrm{c}}^{2} \tau_{y}^{2}} \\
X_{y}^{\prime \prime}\left(\omega_{\mathrm{c}} \tau_{y}\right)=\frac{G_{y}^{\prime \prime}}{\Delta G^{\prime}}=\frac{\omega_{\mathrm{c}} \tau_{y}}{1+\omega_{\mathrm{c}}^{2} \tau_{y}^{2}} .
\end{gathered}
$$

$X_{y}^{*}\left(\omega_{\mathrm{c}} \tau_{y}\right)$ characterizes the phenomenon of relaxationfrequency dispersion of a cell with $y$ contacts and the relaxation time $\tau_{y}$ linked in a colony, while a stress-field with the frequency $\omega_{\mathrm{c}}$ is active. According to Fig. 2a, the real part $X_{y}^{\prime}\left(\omega_{\mathrm{c}} \tau_{y}\right)$, giving the relative strain-energy exhibits a sigmoidal pattern while the relative loss $X_{y}{ }^{\prime \prime}\left(\omega_{\mathrm{c}} \tau_{y}\right)$ goes through a maximum at $\omega_{\mathrm{c}} \tau_{y}=1$ (grey bar). 
Fig. 2 (a) Real part $X_{y}^{\prime}\left(\omega_{\mathrm{c}} \tau_{y}\right)$ and imaginary part $X_{y}^{\prime \prime}\left(\omega_{\mathrm{c}} \tau_{y}\right)$ of the complex relaxation function $X_{y}^{*}\left(\omega_{\mathrm{c}} \tau_{y}\right)$ dependent on $\omega_{\mathrm{c}}$ calculated with Eq. 21 at $\tau_{y}=1.66$ units. The maximum cell number is arrived at the frequency $\omega_{c}=\omega_{\text {cmax }}$. (b) Real and imaginary parts of the complex $\Psi^{*}$ - and $X^{*}$-functions against $t / t_{\mathrm{c}}=\ln \left(\omega_{\mathrm{c}} / \omega_{0}\right)$ at $\tau_{\text {kin }}=10^{6}$ units

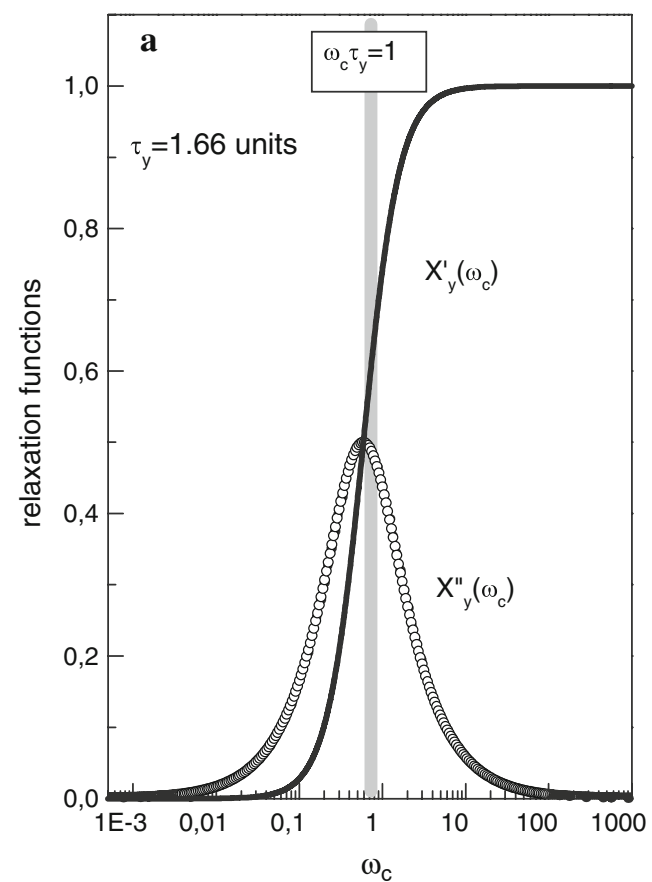

The Growth of a Cell Colony

First, we introduce the normalized ( $p=3$ )-relaxation-mode distribution

$h_{y}\left(\tau_{0 y}\right)=\frac{\Phi\left(\tau_{0 y}, p=3\right)}{\sum_{\tau_{0 y \min }}^{\tau_{0 y \max }} \Phi\left(\tau_{0 y}, p=3\right)}$.

Treating growth of a cell ensemble as a linear process, the total cell number $n_{\mathrm{c}}{ }^{\prime}\left(\omega_{\mathrm{c}}\right)$ is obtained by adding all the fractions $h_{y}\left(\tau_{0 y}\right)$ of differently large cells, each one multiplied by the conjugated factor in Eq. 21. Calling the maximum number of newly formed cells in the grownup colony $\Delta n_{\mathrm{c}}^{\prime}$ and accounting for the existence of the mother cell, the total cell number $n_{\mathrm{c}}^{\prime}\left(\omega_{\mathrm{c}}\right)$ and the corresponding loss $n_{\mathrm{c}}^{\prime \prime}\left(\omega_{\mathrm{c}}\right)$ are equal to

$n_{\mathrm{c}}^{\prime}\left(\omega_{\mathrm{c}}\right)=1+\sum_{y} n_{c y}^{\prime}\left(\omega_{\mathrm{c}}\right)=1+\Delta n_{\mathrm{c}}^{\prime} \sum_{y} h_{y}\left(\tau_{0 y}\right) X_{y}^{\prime}\left(\omega_{\mathrm{c}} \tau_{y}\right)$,

$n_{\mathrm{c}}^{\prime \prime}\left(\omega_{\mathrm{c}}\right)=\sum_{y} n_{\mathrm{cy}}^{\prime \prime}\left(\omega_{\mathrm{c}}\right)=\Delta n_{\mathrm{c}}^{\prime} \sum_{y} h_{y}\left(\tau_{0 y}\right) X_{y}^{\prime \prime}\left(\omega_{\mathrm{c}} \tau_{y}\right)$.

At the lower limit $n_{\mathrm{c}}^{\prime}\left(\omega_{\mathrm{c}}\right)$ is equal to 1 , i.e. the colony develops from a single mother cell. At high frequencies, the maximum cell number comes out to be $n_{\mathrm{cmax}}^{\prime}\left(\omega_{\mathrm{c}}\right)=$ $1+\Delta n_{\mathrm{c}}^{\prime}$. Plotting $n_{\mathrm{col}}^{\prime}\left(\omega_{\mathrm{c}}\right)=n_{\mathrm{c}}^{\prime} / n_{\mathrm{cmax}}^{\prime}$ and $n_{\mathrm{col}}^{\prime \prime}\left(\omega_{\mathrm{c}}\right)=n_{\mathrm{c}}^{\prime \prime \prime} /$ $n_{\mathrm{cmax}}^{\prime}$ against $t / t_{\mathrm{c}}$, Fig. $2 \mathrm{~b}$ illustrates that the width of the dispersion regime embraces the principal contribution according to Eq. 21 (Fig. 2a) plus the additional broadening caused by the existence of the spectrum $h_{y}\left(\tau_{0 y}\right)$ (Eq. 22).

The growth curve $n_{t}^{\prime}(t)$ is obtained by presenting $n_{\mathrm{c}}^{\prime}\left(\omega_{\mathrm{c}}\right)$ in dependence of the time $t$ that is related to $\omega_{\mathrm{c}}$ as formulated in Eq. 19; the width of the dispersion regime depends strongly on $t_{\mathrm{c}}$.

The Growth of Cell Populations

Perfect coordination of all processes is only possible in colonies with a number of cells below a maximum value. A cell population with optimal properties should thus be comprised of $n_{0}$ equivalent subsystems that show qualities similar to a cell colony.

Let a culture experiment begin with $n_{0}$ cells, each one operating as the mother cell of a subsystem. If coordinated growth of the subsystems leads to an affine transformation of the ensemble structure, $n_{0}$ stays constant. In a growing population, the cell number $n_{\mathrm{c}}^{\prime}\left(\omega_{\mathrm{c}}\right)$ and $n_{\mathrm{c}}^{\prime \prime}\left(\omega_{\mathrm{c}}\right)$ are then equal to

$$
\begin{aligned}
& n_{\mathrm{c}}^{\prime}\left(\omega_{\mathrm{c}}\right)=n_{0}\left(1+\Delta n_{\mathrm{c}}^{\prime} \sum_{y} h_{y}\left(\tau_{0 y}\right) X_{y}^{\prime}\left(\omega_{\mathrm{c}} \tau_{y}\right)\right), \\
& n_{\mathrm{c}}^{\prime \prime}\left(\omega_{\mathrm{c}}\right)=n_{0} \Delta n_{\mathrm{c}}^{\prime} \sum_{y} h_{y}\left(\tau_{0 y}\right) X_{y}^{\prime \prime}\left(\omega_{\mathrm{c}} \tau_{y}\right) .
\end{aligned}
$$

with

$n_{\mathrm{c} \text { min }}^{\prime}=n_{0} ; \quad n_{\mathrm{c} \text { max }}^{\prime}=n_{0}\left(1+\Delta n_{\mathrm{c}}^{\prime}\right)$.

Growth curves of populations are simply scaled by the structure factor $n_{0}$. If the number of subsystems is large $\left(n_{0} \gg 1\right)$, the maximum cell number $n_{\mathrm{cmax}}^{\prime}=n_{0}\left(1+\Delta n_{\mathrm{c}}^{\prime}\right)$ may exceed by far the maximum size of a single subsystem $\left(1+\Delta n_{\mathrm{c}}^{\prime}\right)$. Since the maximal number of newly formed cells $\Delta n_{\mathrm{c}}^{\prime}$ is usually very large, the approximation 
$n_{\mathrm{c}}^{\prime}\left(\omega_{\mathrm{c}}\right) \simeq \Delta n_{\mathrm{cc}}^{\prime} \sum_{y} h_{y}\left(\tau_{0 y}\right) X_{y}^{\prime}\left(\omega_{\mathrm{c}} \tau_{y}\right) ; \quad \Delta n_{\mathrm{cc}}^{\prime}=n_{0} \Delta n_{\mathrm{c}}^{\prime}$,

$n_{\mathrm{c}}^{\prime \prime}\left(\omega_{\mathrm{c}}\right) \simeq \Delta n_{\mathrm{cc}}^{\prime} \sum_{y} h_{y}\left(\tau_{0 y}\right) X_{y}^{\prime}\left(\omega_{\mathrm{c}} \tau_{y}\right)$

indicates that all patterns calculated with a constant value of $\Delta n_{\mathrm{cc}}^{\prime}=n_{0} \Delta n_{\mathrm{c}}^{\prime}$ but different combinations of $n_{0}$ and $\Delta n_{\mathrm{c}}^{\prime}$ are identical, i.e. we cannot determine the absolute value of the structure-parameter $n_{0}$ by describing a growth curve with the use of Eq. 24.

The $\Psi^{\prime}$ - and $\Psi^{\prime \prime}$-functions

It seems to be a necessity that growth laws guarantee statistically reliable optimal properties of living systems. The growth logistics given in generally applicable terms should evidence this symmetry. To prove this, we define the $\Psi^{*}$-function by relating $n_{\mathrm{c}}^{\prime}\left(\omega_{\mathrm{c}}\right)$ and $n_{\mathrm{c}}^{\prime \prime}\left(\omega_{\mathrm{c}}\right)$ in Eq. 24 to the maximum cell number $n_{\mathrm{cmax}}^{\prime}=n_{0}\left(1+\Delta n_{\mathrm{c}}^{\prime}\right)$ and by plotting this ratio against the dimensionless variable $t / t_{\mathrm{c}}=\ln \left(\omega_{\mathrm{c}} / \omega_{0}\right)$.

$$
\begin{aligned}
\Psi^{*}\left(\omega_{\mathrm{c}}\right) & =\Psi^{\prime}\left(\omega_{\mathrm{c}}\right)+i \Psi^{\prime \prime}\left(\omega_{\mathrm{c}}\right) \\
\Psi^{\prime}\left(\omega_{\mathrm{c}}\right) & =\frac{n_{0} n_{\mathrm{c}}^{\prime}\left(\omega_{\mathrm{c}}\right)}{n_{0}\left(1+\Delta n_{\mathrm{c}}^{\prime}\right)} \\
& =\frac{1}{1+\Delta n_{\mathrm{c}}^{\prime}}+\frac{\Delta n_{\mathrm{c}}^{\prime}}{1+\Delta n_{\mathrm{c}}^{\prime}} \sum_{y} h_{y}\left(\tau_{0 y}\right) X_{y}^{\prime}\left(\omega_{\mathrm{c}} \tau_{y}\right) \\
\Psi^{\prime \prime}\left(\omega_{\mathrm{c}}\right) & =\frac{n_{0} \Delta n_{\mathrm{c}}^{\prime}}{n_{0}\left(1+\Delta n_{\mathrm{c}}^{\prime}\right)} \sum_{y} h_{y}\left(\tau_{0 y}\right) X_{y}^{\prime}\left(\omega_{\mathrm{c}} \tau_{y}\right) .
\end{aligned}
$$

The structure parameter $n_{0}$ drops out: according to our model, cell populations or colonies should exhibit the same growth characteristics. Since $\Delta n_{\mathrm{c}}^{\prime}$ is large $1 /\left(1+\Delta n_{\mathrm{c}}^{\prime}\right) \approx 0$ and $\Delta n_{\mathrm{c}}^{\prime} /\left(1+\Delta n_{\mathrm{c}}^{\prime}\right) \approx 1$, so that the relations emerge

$$
\begin{aligned}
& \Psi^{\prime}\left(\omega_{\mathrm{c}}\right) \cong \sum_{y} h_{y}\left(\tau_{0 y}\right) X_{y}^{\prime}\left(\omega_{\mathrm{c}} \tau_{y}\right) \\
& \Psi^{\prime \prime}\left(\omega_{\mathrm{c}}\right) \cong \sum_{y} h_{y}\left(\tau_{0 y}\right) X_{y}^{\prime \prime}\left(\omega_{\mathrm{c}} \tau_{y}\right) .
\end{aligned}
$$

$\Psi^{\prime}\left(\omega_{\mathrm{c}} \tau_{y}\right)$, giving the relative strain-energy, exhibits a sigmoidal pattern while the loss $\Psi^{\prime \prime}\left(\omega_{\mathrm{c}} \tau_{y}\right)$ goes through a maximum at $\omega_{\mathrm{c}} \tau_{y}=1$ (Fig. 2b). Since the $(p=3)$ relaxation-mode-spectrum $h_{y}\left(\tau_{0 y}\right)$ is invariant, the growth logistics should be the same for cells of every kind.

The general applicability of the model is easily tested experimentally by checking whether at the same parameter $\tau_{\text {kin }}$, the ratio $n_{\mathrm{c}}^{\prime}\left(\omega_{\mathrm{c}}\right) / n_{\mathrm{cmax}}^{\prime}$ plotted against of $t / t_{\mathrm{c}}$ coincides with the universal $(p=3)-\Psi^{\prime}$-function.

\section{Results}

Eukaryotic Cells

\section{Adherent Mammalian Cells}

The cell number $n_{\mathrm{c}}^{\prime}(t)$ and the individual DNA content of Chinese Hamster Ovary (CHO)-cells were determined by flow cytometry [23]. Each growth curve in Fig. 3a, b starts at the cell number $n_{\text {initial }}$ listed in Table 3 . Since the $n_{\text {initial }}$ cells adjust rapidly optimal conditions, each experimental data set in Fig. 3 can completely be fitted with Eqs. 19 and 24 , using apart from $\tau_{\text {kin }}$ the same set of parameters (Table 3). The growth curves have then the same shape but different positions on the time axis. The position of each pattern is fitted by assigning the kinetic factor to values as shown in Table 3. According to Fig. 3e, the kinetic factor increases nearly linear with $n_{\text {initial }}$. Apparently, at $t=0$ the virtual age of the patterns increases with the initial concentration of the nutrient.

Since the growth curves show altogether the same shape, a master curve is obtained by "simply shifting" each original data set along the time-axis until the pattern in Fig. 3c, d results that corresponds to a theoretical curve calculated at $\tau_{\text {kin }}=4 \times 10^{-6} \mathrm{~h}$.

Each dotted line in Fig. 3b-d describes the energy loss during growth. Unfortunately, the dissipation heat has not been measured until now. Yet, the calculation reveals that in the regime of exponential growth (in Fig. 3d between 30 and $60 \mathrm{~h}) \ln \left(n_{\mathrm{c}}^{\prime}(t)\right)$ and $\ln \left(n_{\mathrm{c}}^{\prime \prime}(t)\right)$ converge more or less, suggesting that a network-like structure is developing as in a gel [24].

\section{The Growth of a Cell Colony}

Because of being unable to determine the absolute number $n_{0}$ of subsystems by fitting a growth curve (Eq. 24), we studied growing colonies of adherent A549 human lung cancer cells. Cells from an exponentially growing culture were counted with a flow cytometer. They were seeded in $25 \mathrm{~cm}^{2}$ culture vessels at approximately ten cells in $10 \mathrm{ml}$ nutrient and incubated for up to 3 weeks. After 7 and 14 days, cultures were fed with fresh medium or were stained to determine cell numbers in the colonies (see the legend of Fig. 4a).

Micrographs from different times are depicted in Fig. 4. Calculations with the use of Eqs. 19 and 24 reproduce the data satisfyingly (Fig. 5a, b). When replacing the nutrient repeatedly, marginal extra-fluctuations of data points are observed occasionally, may be caused by the technical interventions. The protracted convergence to a final maximum cell number may be due to loosely packed cells on 
Fig. 3 (a) Experimentally determined number of CHO-cells cultured from the initial cell concentrations $n_{\text {initial }}$ as indicated [23]. The solid lines are computed with Eqs. 19 and 24 and the parameters in Table 3. The upper limit of the mode spectrum is equal to $\ln \left(\tau_{0 y \text {.max }}\right)=12.7$, i.e. relaxation mode distribution and the universal cell size distribution exhibit the same shape (Eq. 15). (b) Semilogarithmic plot of the original data and the theoretical curves in (a). (c) Master-curve for $n_{0}=10^{5}$ cells. (d) Logarithmic plot of the data in (c). (e) $\tau_{\text {kin }}$ against $n_{\text {initial }}$
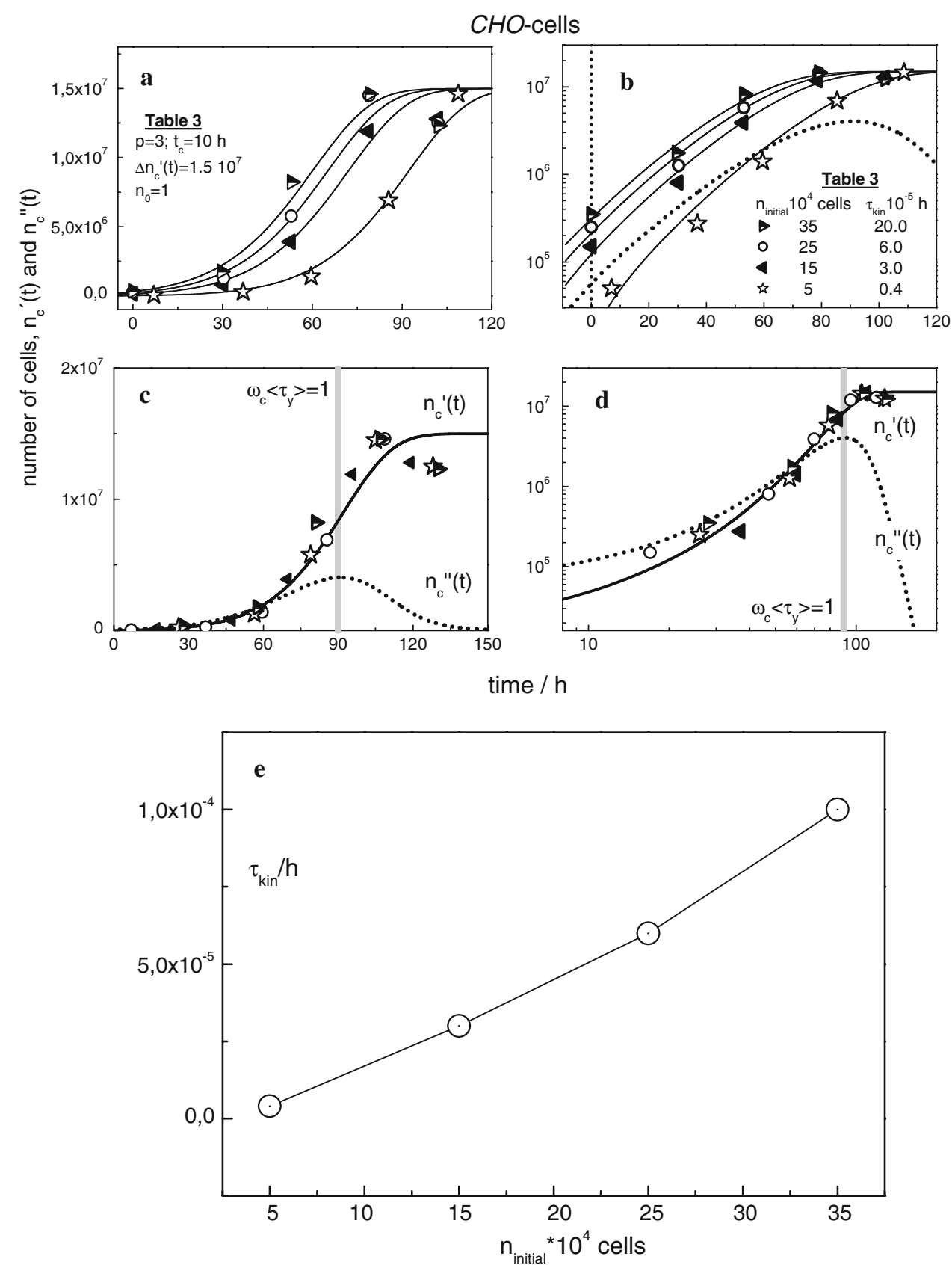

the boundary (arrow in Fig. 4c) where occasional cell divisions may occur. Still, the estimated cell number in a 3week-old colony $(\approx 650,000)$ significantly lags behind the 2-5 Mio cells obtained in the same culture vessel within one week after seeding $10-50,000$ cells.

\section{Cell Populations}

Consistent with the model's predictions, we succeed in describing the growth pattern of a population by using apart from $n_{0}$ and $\tau_{\text {kin }}$ the same parameters as in the preceding section. Within the limits of accuracy, the plots in
Fig. 5c, d demonstrate the satisfying fit achieved with Eqs. 19 and 24 . Setting $\Delta n_{\text {colmax }}^{\prime}$ equal to $6.5 \times 10^{5}$ cells, the population embracing $n_{\text {cmax }}^{\prime}=1.17 \times 10^{7}$ cells should be formed by $n_{0}=n_{\text {cmax }}^{\prime} / n_{\text {colmax }}^{\prime}=18$ subsystems linked in a network.

Showing the growth curve of a colony and of a population at the same position on the time axis (Fig. 5e), the remarkable similarity demonstrates that the growthlogistics in populations and colonies is identical. The subsystems in cell populations seem to exhibit about the same properties as colonies. According to Fig. $5 f$ represented in terms of the dimensionless variables 


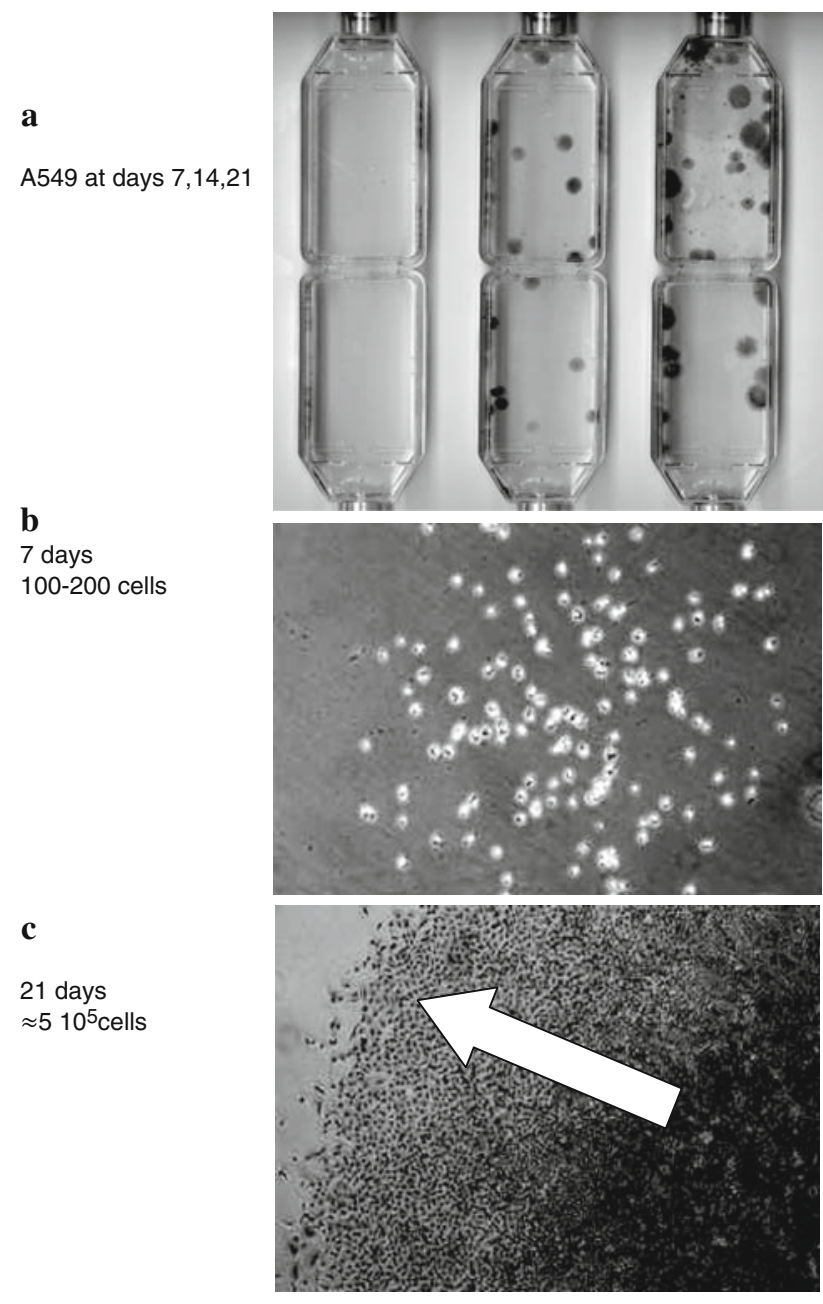

Fig. 4 A 549 cells were seeded at 10 cells $/ 10 \mathrm{ml}$ in $25 \mathrm{~cm}^{2}$ culture flasks. They were incubated for 7, 14 and 21 days, respectively (a). After 7 and 14 days, cultures were fed with fresh medium or were stained to determine cell numbers in the colonies. (a) After 1 week, colonies were hardly visible. Upon microscopic inspection, their cell numbers ranged around 200 (b). Counting under the microscope the cells along the diameter of the three weeks old colonies (c), the maximum cell number was extrapolated to $\approx 5 \times 10^{5}$ cells; the arrow should point to the declining cell-density in the ensemble boundaries

$\left(n_{\mathrm{c}}^{\prime} / n_{\mathrm{cmax}}^{\prime} ; t / t_{\mathrm{c}}\right)$, all the data coincide with the $(p=3)-\Psi^{\prime}-$ function, proving the validity of our concept. If in correspondence with Pelling et al. [12] the frequency $\omega_{0}$ of signals emitted by a single cell (Eq. 17) is set equal to $\omega_{0} \approx 1 \mathrm{kHz}$ the values of $\omega_{\mathrm{c}}$ indeed match the range of acoustic waves in liquids at room temperature [25].

Most likely, relaxation-frequency-dispersion limits the size of a colony to a maximum cell number, usually smaller than the maximal cell number of a grown-up population. As an optimal structure, any cell population should form an assembly of equivalent subsystems into a transient network.
The Influence of the Nutrient

We regard the growth process in $\mathrm{CHO}$-cultures, started with $10^{5}$ cells at $100 \%$ nutrient concentration in the first culture (C1) and 30\% in the second (C2). With Eqs. 19 and 24 and the same value of $t_{\mathrm{c}}=0.55 d$ both experimental patterns in Fig. 6 are fitted fairly well. Hence, the period of cell cycles does not depend on the amount of nutrient that is available. For different culture media, remarkable modifications are observed [26].

The maximum cell number in the (C1)-pattern $n_{\mathrm{cmax}}^{\prime}=n_{01}\left(1+\Delta n_{\mathrm{c}}^{\prime}\right)$ is equal to $1.5 \times 10^{7}$ cells. Assigning $\Delta n_{\mathrm{c} 1}^{\prime}=\Delta n_{\mathrm{c} 2}^{\prime}$ of the subsystems to $6.5 \times 10^{5}$ cells (as observed in the previous experiments), the (C1)-population should contain $n_{01}=n_{\mathrm{cmax}}^{\prime} / \Delta n_{\mathrm{c} 1}^{\prime}=16.1$ subsystems. If the ratio $n_{02} / n_{01}$ agrees with the ratio of the nutrient concentrations 30/100 the number of subsystems in the (C2)population drops to $n_{02}=6.9\left(n_{\mathrm{cmax}}^{\prime}=6.9\left(1+\Delta n_{\mathrm{c}}^{\prime}\right)=\right.$ $4.5 \times 10^{6}=0.30 \times 1.5 \times 10^{7}$ cells $)$. When a culture experiment is started, the initial concentration of nutrient seems to exercise a crucial influence on the optimal ensemble structure that is adjusted, rapidly.

In order to obtain in the calculation at $t=0$ exactly $n_{\text {initial }}=10^{5}$ cells, the kinetic factor, must be assigned to the different values $\tau_{\mathrm{kinC} 1}=3.5 \times 10^{-5} d$ and $\tau_{\mathrm{kinC} 2}$ to $1.15 \times 10^{-4} d$. At $t=0$ the virtual age of (C2)-subsystems is about thrice the one of (C1)-subsystems. Since at $t=0$, the size of (C2)-subsystems is then about 3.34 times the size of $(\mathrm{C} 1)$-patterns, in both cultures the cell number is equal to $n_{\mathrm{c}}^{\prime}=n_{\text {initial }}=10^{5}$ cells.

In terms of our model, the number of mother cells $n_{0}$ seems to increase with the concentration of the nutrient. If the subsystem's ensemble structure undergoes then at $n_{0}=$ const a growth induced affine transformation, the underlying processes run off on optimal conditions. Of course, as long as the product of $n_{0} \Delta n_{\mathrm{c}}^{\prime}$ remains constant, very intricate growth processes are theoretically equivalent.

\section{The $\Psi^{\prime}$-function and the Cell Cycle}

During the cell cycle, every growing cell passes through the phases shown in Fig. 7. The fractions $f_{\mathrm{G} 1}(t), f_{\mathrm{S}}(t)$ and $f_{\mathrm{G} 2 \mathrm{M}}(t)$, defined in this figure, are deduced from flow cytometric measurements of the DNA content [23]. According to Fig. 8, in the grown-up population about $95 \%$ of the cells are in the G1-phase, virtually no cells in the S-phase, and about $5 \%$ of the cells in the G2M-phase. The fraction $f_{\mathrm{G} 1}(t)$ and the total cell number (dotted line) run parallel, i.e. $f_{\mathrm{G} 1}$ grows analogous to the $(p=3)-\Psi^{\prime}\left(\omega_{\mathrm{c}}\right)$ function (Eq. 26). During growth, the cell cycle distribution should then show the characteristics 
Fig. 5 (a) $\square \bigcirc$ data of various A549 colonies, dependent on time; the mean values. The solid line is calculated with Eqs. 19 and 24. (b) Logarithmic plot of (a). (c) Cell number of A549 populations; the solid line is computed with Eqs. 19 and 24. (d) Logarithmic plot of (c). (e) Comparison of the logarithmic growth curves of a colony and a population. (f) The universal $\Psi^{\prime}$-function; for $\omega_{0}=10^{3} \mathrm{~Hz}$ [12] the frequency $\omega_{\mathrm{c}}$ falls in the range of acoustic signals in liquids at temperatures of $20-25^{\circ} \mathrm{C}[25]$
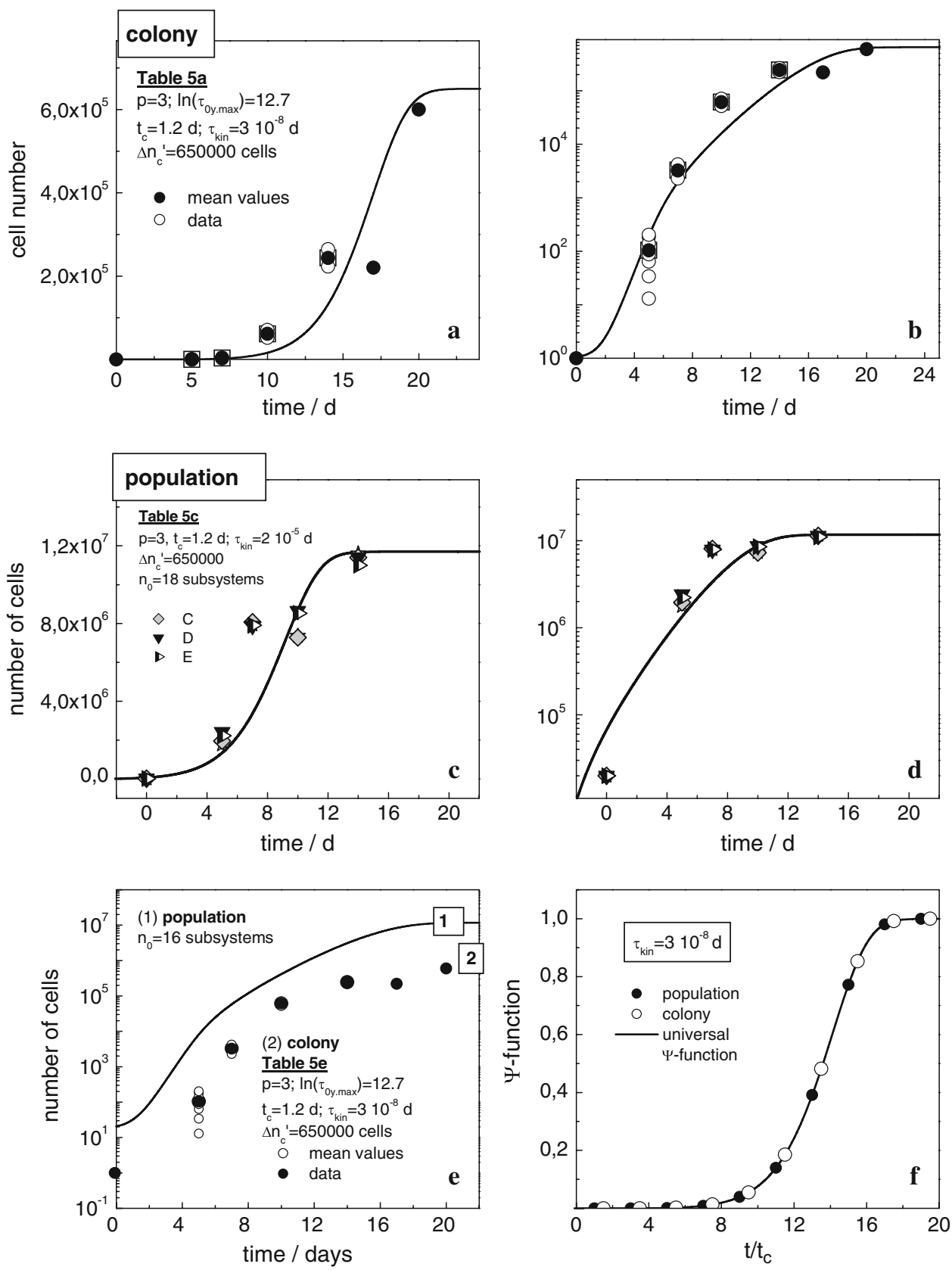

$f_{\mathrm{G} 1}=\varepsilon_{\mathrm{G} 11} \Psi^{\prime}\left(\omega_{\mathrm{c}}\right)+\varepsilon_{\mathrm{G} 12}$

$f_{\mathrm{S}}=\varepsilon_{\mathrm{S}}\left(1-\Psi^{\prime}\left(\omega_{\mathrm{c}}\right)\right)$

$f_{\mathrm{G} 2 \mathrm{M}}=\left(\varepsilon_{\mathrm{G} 2 \mathrm{M}}-\varepsilon_{\mathrm{G} 2 \mathrm{M} 0}\right)\left(1-\Psi^{\prime}\left(\omega_{\mathrm{c}}\right)\right)+\varepsilon_{\mathrm{G} 2 \mathrm{M} 0}$.

When a culture experiment is started, and optimal process conditions are adjusted, the distribution is characterized by the parameters $\varepsilon_{11}, \varepsilon_{12}$ and $\varepsilon_{\mathrm{S}}\left(\varepsilon_{\mathrm{G} 2 \mathrm{M}}=1-\varepsilon_{\mathrm{G} 12}-\varepsilon_{\mathrm{S}}\right)$. In the grown-up colony $\varepsilon_{\mathrm{G} 2 \mathrm{M} 0}$ gives the fraction of cells in the G2M-phase. The solid lines in Fig. 8 are calculated with the use of Eqs. 19 and 27 and the parameters in Tables 3a,b and 8 (only $\tau_{\text {kin }}$ is slightly reduced: $\tau_{\text {kin }}=2.5 \times 10^{-6} \mathrm{~h} \Rightarrow$ $\tau_{\text {kin }}=7 \times 10^{-7} \mathrm{~h}$ ).
During growth, the fraction of G1-cells, and possibly the transitions into the $\mathrm{S}$-phase, are directly related to the phenomenon of relaxation-frequency-dispersion. On the biochemical level, cell cycle checkpoints with related regulatory proteins [27] are the means to translate the processes represented by the stress field into a frequencydependent cellular response.

\section{Human Melanocytes}

Human melanocytes exhibit growth curves as shown in Fig. 9 [28]. For technical reasons, the initial cell concentrations vary systematically, in each case collecting data 


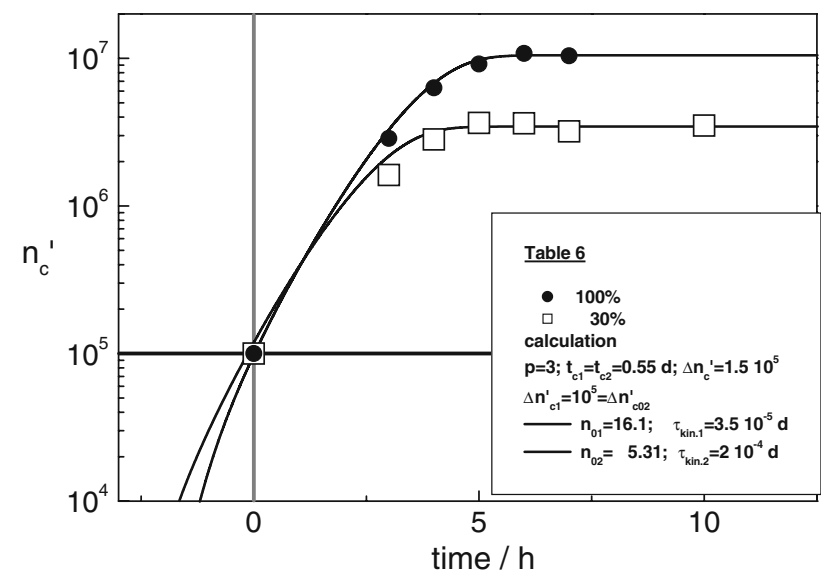

Fig. 6 Growth curves of K562 leukaemic cells, each one started with $10^{5}$ cells in presence of $100 \%$ ( ), respectively, $30 \%(\square)$ nutrient. The solid lines are computed with Eqs. 19 and 24, using the parameters in Table 6

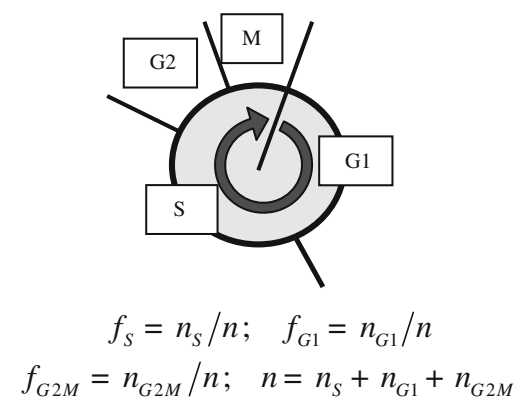

Fig. 7 The cell cycle: G1-phase: cells contain the standard amount of DNA. During the S-phase, the DNA is replicated. G2-phase: preparation for division, accomplished in mitosis, M. The definition of the fractions $f_{\mathrm{G} 1}, f_{\mathrm{S}}$ and $f_{\mathrm{G} 2 \mathrm{M}}$ depicted at the bottom, gives the relative number of cells in the phases as indicated

over 12 culture days. The individual patterns can be fitted with Eq. 19 and 24 by using (apart from $\tau_{\text {kin }}$ ) always the same parameters (Table 9). In analogy to the precedent example of CHO-cell patterns, $\tau_{\text {kin }}$ is set to those individual values that are indicated with each curve. The conspicuous resemblance of the growth curves is a precondition to construct the master curve in Fig. 9b, c by shifting each data set along the time axis. In agreement with our model, rheological simplicity is again proven to typify the growth process of cell cultures.

\section{Murine Leukaemia Cells and S. cerevisiae}

With the use of Eqs. 19 and 24, we also come to a full reproduction of the growth curves of murine leukaemia cells [29] (Fig. 10) and S. cerevisiae [30] (Fig. 11).

\section{Prokaryotic Cells}

\section{Escherichia coli}

Finally, we discuss the modification of the cell size distribution structure during growth. Figure 12 shows the growth curve of an E. coli population measured by Schlote [9]. The growth curve can be fitted with the use of Eqs. 19 and 24.

The inserts in Fig. 12 present the cell size distributions determined at the time points 3-6 along the growth curve [9]. The width of the distributions decreases in the course of time. Equation 9 allows us to reproduce these patterns, and to identify them as members of the universal $\Phi(\eta$, $p=3$ )-class (Fig. 1).

An optimal structure of a growing cell ensemble is installed even in presence of increasing constraints, originating from the formation of a transient cellular network. According to Fig. 13, all the values of $\beta \Delta u_{0}(t)$ fall below 0.023; compared with the mean thermal energy $k_{\mathrm{B}} T$ the contact energy of ICs is relatively small. Hence, ever-present structural fluctuations support even complicated molecular rearrangements during relaxation.

Discussing the changes of the reaction energy, we should keep in mind that at a given time this parameter is the same for all ICs irrespective of the size of the incorporating cell. At the beginning of a culture experiment $\beta \Delta u_{0}(t)$ usually drops within $\Delta t \approx t_{\mathrm{c}}$ to optimized patterns with a minimal reaction energy, increasing then in correspondence with the growth process. $\Delta u_{0}(t)$ is thus described by

$$
\begin{aligned}
\Delta u_{0}(t) & =\chi_{1} \sum_{y} \exp \left\{-\chi_{2} t / \tau_{y}\right\}+\Delta u_{\mathrm{c}}+A_{0}(t) ; \\
A_{0}(t) & =\zeta \Psi^{\prime}\left(\omega_{\mathrm{c}}\right) \geq 0 .
\end{aligned}
$$

The exponential term accounts for the rapid initial adaptation immediately after the experiment has been started. Afterwards, the density of constraints grows according to $A_{0}(t)$, i.e. controlled by the relaxationfrequency-dispersion.

According to the underlying concept, the mean size of the cells $\langle y\rangle$ should decrease during growth. From Eq. 9 $<y>$ is deduced to be (7)

$<y(t)>\Delta u_{0}(t)=(p+1) k_{\mathrm{B}} T=$ const.

The above relation causes the ratio of $\langle y\rangle_{\text {min }} /\langle y\rangle_{\text {max }}$ to be equal to $\Delta u_{0 \max } / \Delta u_{0 \min }$. With the data of Fig. 13 we arrive at

$<y>_{\min } /<y>_{\max }=\Delta u_{0 \max } / \Delta u_{0 \min } \cong 0.37$.

The mean size of E.coli cells in a grown-up population is only about $37 \%$ of the initial value; these relatively small 
Fig. $8 f_{\mathrm{G} 1}(t), f_{\mathrm{S}}(t)$ and $f_{\mathrm{G} 2 \mathrm{M}}(t)$ of a growing $\mathrm{CHO}$-population [23]. Experimental data $\nabla \bigcirc$ is $\triangleleft$. The solid lines are computed with Eq. 26, the parameters in Tables 8 and 3 . The dotted line gives the number of cells $n_{\mathrm{c}}^{\prime}(t)$ calculated with Eqs. 19 and 24 and the parameters as in Table 3, with exception of the reduced value of the kinetic factor $\left(\tau_{\text {kin }}=2.5 \times 10^{-6} \mathrm{~h} \Rightarrow\right.$ $\left.\tau_{\text {kin }}=7 \times 10^{-7} \mathrm{~h}\right)$
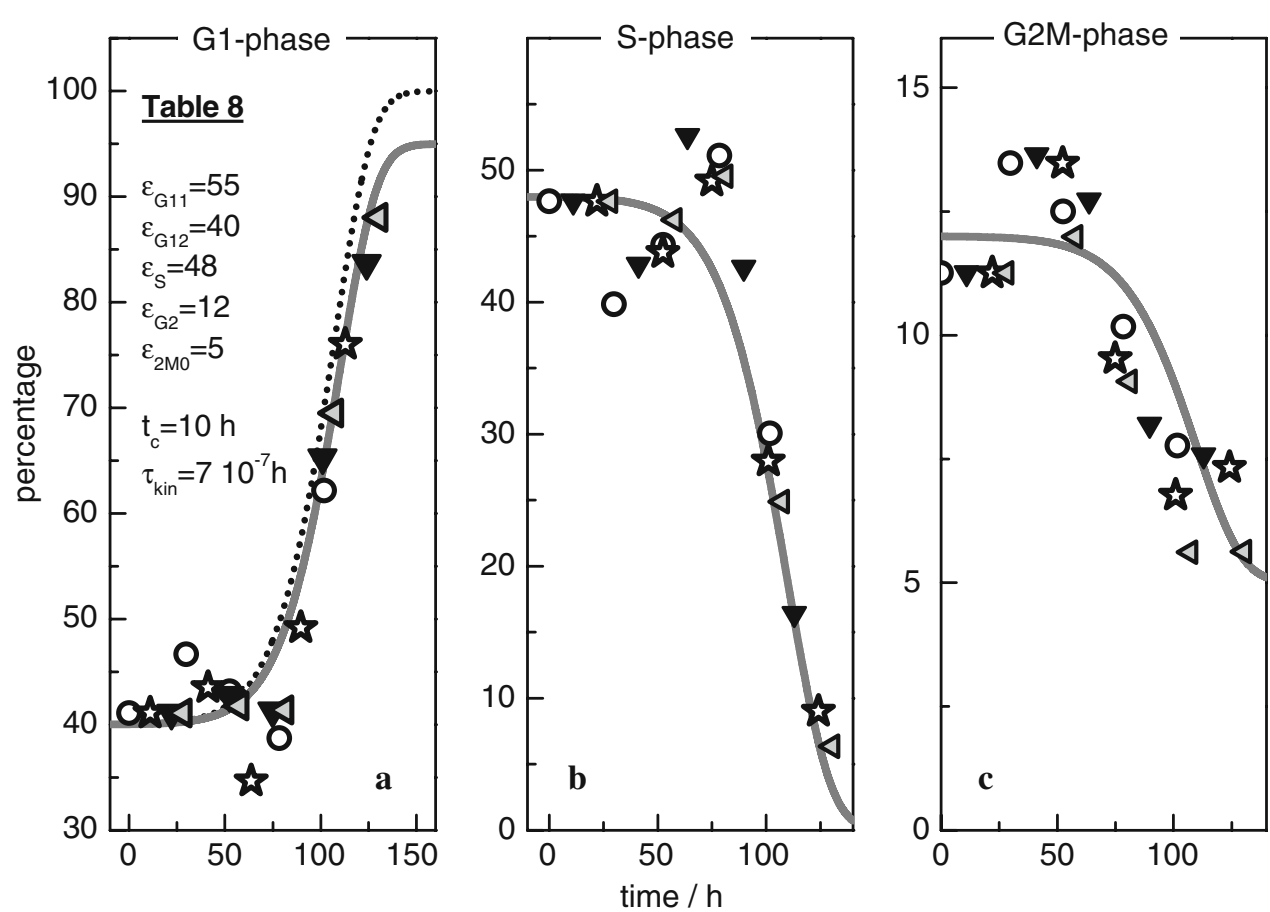

Fig. 9 (a) Cell density $n_{\mathrm{c}}^{\prime}(t)$ of adherently growing human melanocytes [28].

Measurements over 12 days have been started at various cell concentrations. The solid lines are computed with Eqs. 19 and 24 , the individual values of $\tau_{k i n}$ are indicated with each curve.

(b) The master curve.

(c) Logarithmic plot

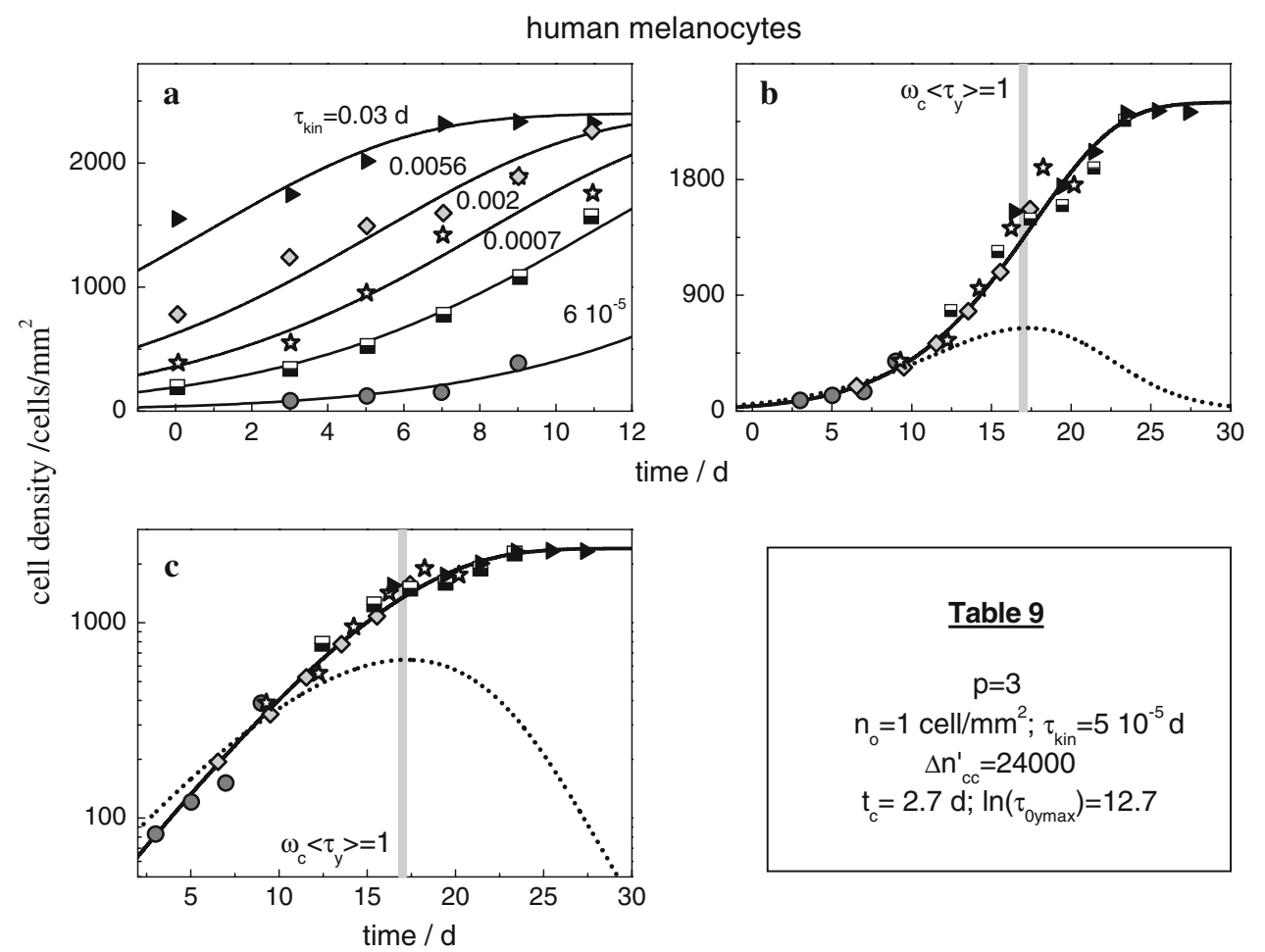

cells have to stay mainly in a G1-like state (see above). It seems that the initiation of DNA-replication is directly related to the conditions set up by the relaxation-frequencydispersion.

\section{Concluding Remarks}

Optimal growth of cell ensembles requires the balance of energy and entropy (according to Eq. 4). This implies a 
Fig. 10 (a, b) Linear and logarithmic plot of the number of cells per $\mathrm{ml}$ of L1210 murine leukaemia cells according to [29]; $\triangleright, o$, s; data, solid lines $n_{\mathrm{c}}^{\prime}(t)$, and dotted lines $n_{\mathrm{c}}{ }^{\prime \prime}(t)$ computed with Eqs. 19 and 24 and the parameters in Table 10

Fig. 11 (a, b) Linear and semilogarithmic plot of $n_{\mathrm{c}}^{\prime}(t)$ and $n_{\mathrm{c}}^{\prime}(t)$-curves of S.cerevisiae according to [30]. $\bigcirc$ data, solid and dotted lines computed with Eqs. 19 and 24 and the parameters in Table 11
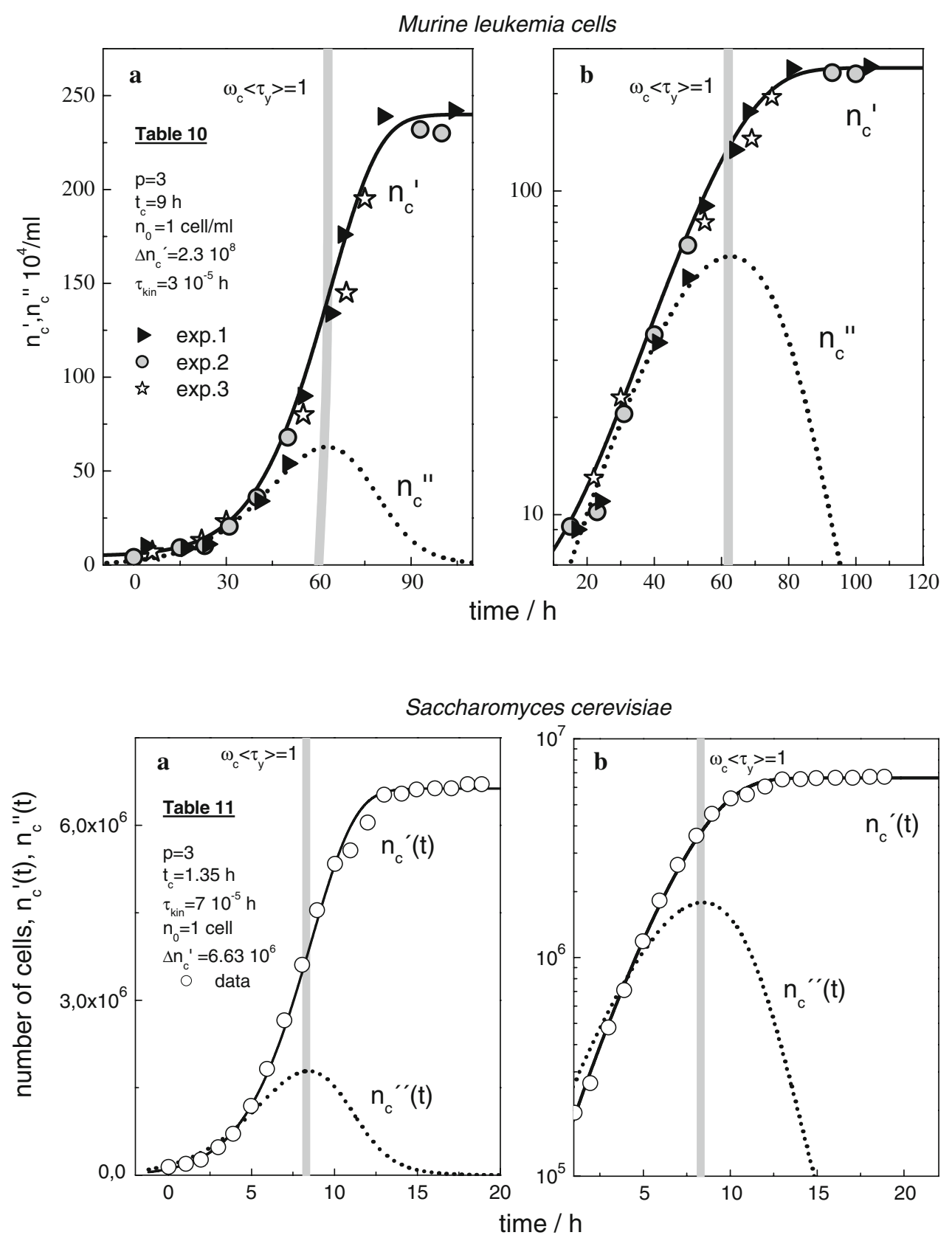

structure-dynamics interrelation as formulated in the increment model. The compulsory dynamics is perpetuated by thermally driven molecular rearrangements of the liquid-like fluctuating cytoplasm. As part of the controlled process of growth and cell division these rearrangements are tantamount to a relaxation process which enables optimization of the individual cell as well as of the whole cell ensemble. The universal validity of the $(p=3)$-cell size distribution confirms this principal characteristics.

Our description of the growth of cell ensembles shows that complete co-ordination is accomplished under the following premises: with an individual cell size-dependent relaxation time, each cell acts as a largely independent visco-elastic element linked in an ensemble characterized by a universal relaxation mode distribution. All cells participate in an all-embracing signal-response system, always related to a thermodynamically defined state of reference, an appropriate situation specificity and an ample adaptability to complex global conditions of the ensemble.

Besides the various known biochemical processes which are governed by diffusion or active molecular translocation, intercellular communication might rely on the much faster acoustic signals as observed by Pelling et al. [12], emitted and perceivable by every growing cell in a population. The overlaid signals seem to be represented by a quasi-periodic field that allows for a collective response via 


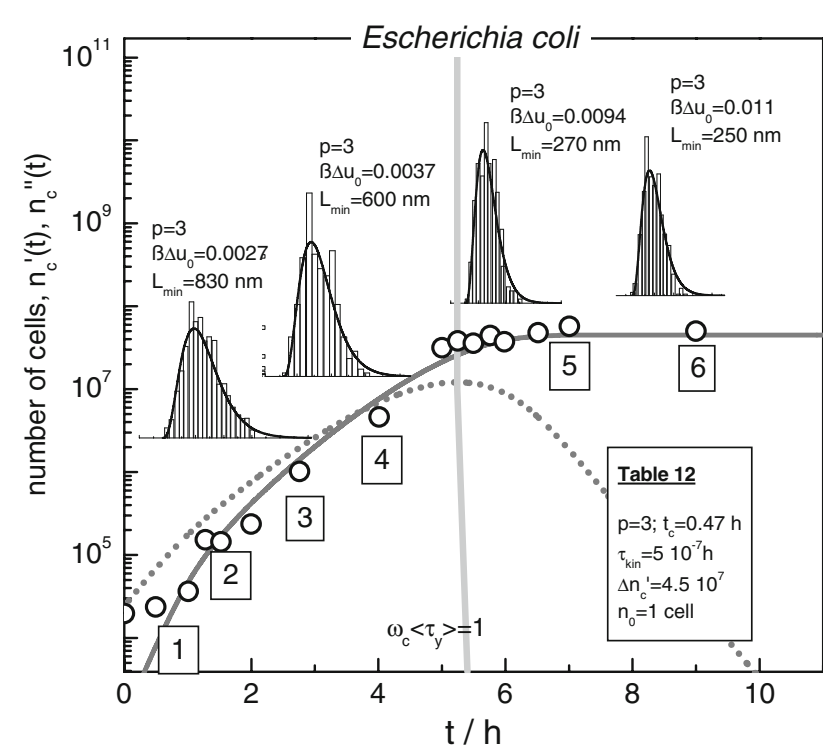

Fig. 12 The growth of Escherichia coli populations according to Schlote [9] ( $\bigcirc$ number of Escherichia cells). The solid line $\left(n_{\mathrm{c}}^{\prime}(t)\right)$ and the dotted line $\left(n_{\mathrm{c}}^{\prime \prime}(t)\right)$ are computed with Eqs. 19 and 24 and the parameters in Table 12. $n_{\mathrm{c}}^{\prime \prime}(t)$ is maximal at $\left\langle\tau_{y}\right\rangle \omega_{\mathrm{c}}=1$, indicated by a grey bar. The inserts (3), (4), (5), (6) show stationary size distributions. The solid lines are computed with Eq. 9, putting $\beta \Delta u_{0}$ and the birth length $L_{\min }$ to the values as indicated $\left(L_{\min }=y / q_{0}\right.$, $y=$ volume, $q_{0}$ as cross-section of the rod-shaped cells) [7]

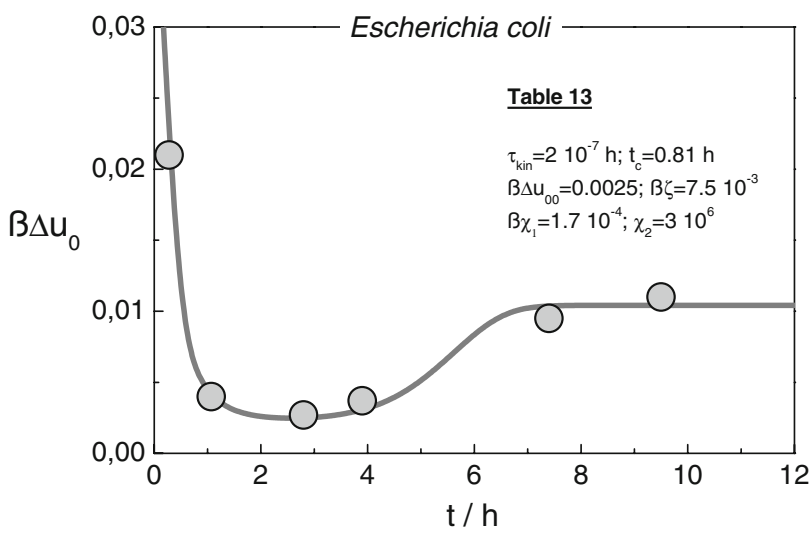

Fig. $13 \beta \Delta u_{0}(t)$ determined from the description of the cell size distributions at the time points $1-6$ in Fig. 12 by using Eq. 9; the solid line is computed with Eq. 28 and the parameters in Table 13

relaxation. This relaxation occurs at the restricted rate of the molecular reconfigurations, while the signal sequence accelerates steadily with the growing cell number. The resulting phenomenon of relaxation-frequency-dispersion then becomes the central controlling and limiting mechanism of the growth process. It is important to note that it

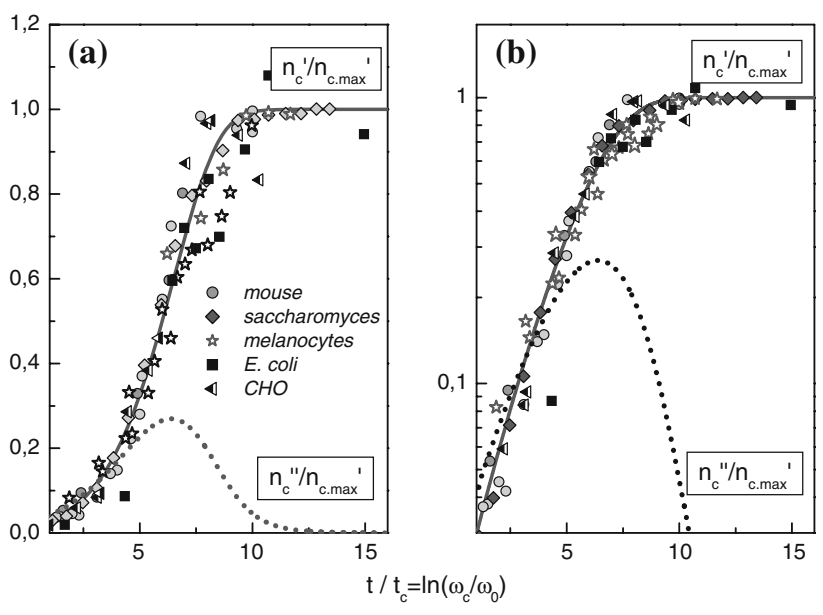

Fig. 14 (a) The growth curves of the systems studied here in terms of the dimensionless variables $n_{\mathrm{c}}^{\prime} / n_{\mathrm{cmax}}^{\prime}$ and $t / t_{\mathrm{c}}=\ln \left(\omega_{\mathrm{c}} / \omega_{0}\right)$. The solid line is the $(p=3)-\Psi^{\prime}$-function, calculated with Eq. 26 plotted then against $t / t_{\mathrm{c}}$ at the kinetic factor of $\tau_{\text {kin }}=6 \times 10^{-5}$ units and $\ln \left(\tau_{\text {Oymax }}\right)=12.7$; the dotted line gives the $\Psi^{\prime \prime}$-function (b) the logarithmic plot

also enables the organization of cells in statistically equivalent optimized tissue-like structures. The process is identical in cells of all kinds as it always follows the same rules. The highly co-operative events are the first to be blocked. Therefore, they typify the topological features of the structure of every grown-up cell ensemble. However, less complex rearrangements take place under optimal conditions, still.

The universal character of our concept is compellingly demonstrated in Fig. 14: independent of intrinsic factors like cell shape or environmental parameters given by culture conditions, the $(p=3)$-version of the $\Psi^{\prime}$-function typifes the topologically uniform growth behaviour of cell ensembles of widely different species. The same laws should likely control the growth and development of higher, i.e. multicellular organisms, as well.

Hence, throughout growth of cell colonies or populations, the outlined relations define an invariable logistic framework that guarantees reproducible structural properties, installed under nearly optimal process conditions, independent of exogenous or endogenous factors. Different genetic patterns can thus develop on an individual as well as on an evolutionary scale. These results may allow us to extend the Taylor and Weibel principle [31]: "Biological structures are formed, controlled by a general growth logistics to meet but not to exceed maximum requirements".

Acknowledgements We have had stimulating discussions with H. Ringsdorf (Mainz) and H. Gruler (Ulm). We are grateful for getting valuable information by J.P. Spatz (MPI Metal Research, Stuttgart). 


\section{Appendix}

In order to describe the relaxation process of a cell with $y$ contacts by a single exponential function, we introduce the reaction variable $\xi_{y}$. Under isothermal-isobaric conditions, the differential of the Gibbs-potential $d \mathrm{~g}_{y}$ is equal to [15] $\left(d \mathrm{~g}_{y}\right)_{T, p}=-A_{y} d \xi_{y}$.

The affinity $A_{y}>0$ measures the distance from optimal conditions $\left(A_{y}=\right.$ const $)$. At small distances from this state of reference, the reaction-rate obeys the differential equation

$\dot{\xi}_{y}=a A_{y} ; \quad A_{y}=$ const $>0 ; \quad a=$ const $>0$.

$a=$ const $>0$ is Onsager's coefficient. Neglecting enthalpy contributions

$$
\begin{aligned}
-A_{y}\left(\xi_{y}\right) & =\left(\frac{\partial \mathrm{g}_{y}\left(\xi_{y}\right)}{\partial \xi_{y}}\right)_{T, p} \\
& =\left(\frac{\partial h_{y}\left(\xi_{y}\right)}{\partial \xi_{y}}\right)_{T, p}-T\left(\frac{\partial s_{y}\left(\xi_{y}\right)}{\partial \xi_{y}}\right)_{T, p} \\
& \approx-T\left(\frac{\partial s_{y}\left(\xi_{y}\right)}{\partial \xi_{y}}\right)_{T, p},
\end{aligned}
$$

we are led to

$\dot{\xi}_{y}=a T\left(\frac{\partial s_{y}\left(\xi_{y}\right)}{\partial \xi_{y}}\right)_{T, p}$.

Defining the entropy $s_{y}\left(\xi_{y}\right)$ as function of the microstates $\Omega_{y}$ in a cell with $y$ contacts by $s_{y}\left(\xi_{y}\right)=\mathrm{k}_{\mathrm{B}} \ln \left(\Omega_{y}\left(\xi_{y}\right)\right)$ Eq. 31 reads

$\dot{\xi}_{y}=a k_{\mathrm{B}} T\left(\frac{\partial \ln \Omega_{y}\left(\xi_{y}\right)}{\partial \xi_{y}}\right)_{T, p}=\frac{a k_{\mathrm{B}} T}{\Omega_{y}\left(\xi_{y}\right)}\left(\frac{\partial \Omega_{y}\left(\xi_{y}\right)}{\partial \xi_{y}}\right)_{T, p}$.

At a small distance from the optimal growth conditions, the approximation should be appropriate

$$
\begin{gathered}
\Omega_{y}\left(\xi_{y}\right) \simeq \Omega_{0 y}+\left(\frac{\partial \Omega_{y}\left(\xi_{y}\right)}{\partial \xi_{y}}\right)_{0} \xi_{y}+\frac{1}{2}\left(\frac{\partial^{2} \Omega_{y}\left(\xi_{y}\right)}{\partial \xi_{y}^{2}}\right)_{0} \xi_{y}^{2} \\
\left(\frac{\partial \Omega_{y}\left(\xi_{y}\right)}{\partial \xi_{y}}\right) \simeq\left(\frac{\partial \Omega_{y}\left(\xi_{y}\right)}{\partial \xi_{y}}\right)_{0}+\left(\frac{\partial^{2} \Omega_{y}\left(\xi_{y}\right)}{\partial \xi_{y}^{2}}\right)_{0} \xi_{y}
\end{gathered}
$$

and Eq. 32 is then

$$
\begin{aligned}
\dot{\xi}_{y} & +a \frac{k_{\mathrm{B}} T}{\Omega_{0 y}}\left[\left(\frac{\partial \Omega_{y}\left(\xi_{y}\right)}{\partial \xi_{y}}\right)_{0}-\left(\frac{\partial^{2} \Omega_{y}\left(\xi_{y}\right)}{\partial^{2} \xi_{y}}\right)_{o}\right] \xi_{y} \\
& =a \frac{k_{\mathrm{B}} T}{\Omega_{0 y}}\left(\frac{\partial \Omega_{y}\left(\xi_{y}\right)}{\partial \xi_{y}}\right)_{0} .
\end{aligned}
$$

Introducing the phenomenological relaxation time $\tau_{y}$ $\dot{\xi}_{y}+\frac{1}{\tau_{y}} \xi_{y}=a \frac{k_{\mathrm{B}} T}{\Omega_{0 y}}\left(\frac{\partial \Omega_{y}\left(\xi_{y}\right)}{\partial \xi_{y}}\right)_{0}$,

we arrive at

$\tau_{y}=\frac{\Omega_{0 y}}{a k_{\mathrm{B}} T\left[\left(\partial \Omega_{y}\left(\xi_{y}\right) / \partial \xi_{y}\right)-\partial^{2} \Omega_{y}\left(\xi_{y}\right) / \partial^{2} \xi_{y}\right]_{0}}=\frac{\Omega_{0 y}}{a k_{\mathrm{B}} T \mu}>0$

$\mu=\left[\left(\frac{\partial \Omega_{y}\left(\xi_{y}\right)}{\partial \xi_{y}}\right)-\frac{\partial^{2} \Omega_{y}\left(\xi_{y}\right)}{\partial^{2} \xi_{y}}\right]_{0}$.

It is convenient to define the relaxation time $\tau_{y}$ as the product [18]

$\tau_{y}=\tau_{0 y} \tau_{\text {kin }}$

The mode- and the kinetic-factors are then equal to

$\tau_{0 y}=\Omega_{0 y} ; \quad \tau_{\text {kin }}=\frac{1}{a k_{\mathrm{B}} T \mu}$.

\section{References}

1. Alberts, B., Bray, D., Johnson, A., Lewis, J., Raff, M., Roberts, K., \& Walter, P. (1997). Essential cell biology (p. 1). New York, London: Garland.

2. Gompertz, B. (1825). The nature of the function expressive of the law of human mortality, and on a new model of determining the value of life contingencies Philosophical Transactions of the Royal Society, 2, 513.

3. Bertalanaffy, V. L. (1957) Quantitative laws in metabolism and growth. The Quarterly Review of Biology, 32, 217.

4. Scharf, I. H. (1980). In W. Beier \& R. Rosen (Eds.), Wachstum der Medizin. Biophysikalische Grundlagen (2 edn., pp. 62-131). Fischer Verlag.

5. Banavar, J. R., Damuth, J., Maritan, A., \& Rinaldo, A. (2002). Modelling universality and scaling. Nature, 420, 626.

6. Kilian, H. G., Kemkemer, R., \& Gruler, H. (2002). Universality and individuality as complementary factors to optimise and resproduce cell populations. Colloid Polymer Science, 280, 661.

7. Kilian, H. G., Gruler, H., \& Kemkemer, R. (2004). Relaxation processes during cell growth. Progress in Colloid Polymer Science, $125,198$.

8. Kilian, H. G., Gruler, H., Bartkowiak, D., \& Kaufmann, D. (2005). Stationary cell size distributions and mean protein chain length distributions of Archaea, Bacteria and Eurolaraotes described with an increment model in terms of irreversible thermodynamics. European Physics Journal E: Soft Mater, 17, 307.

9. Schlote, F. W. (1961). Über den Feinbau von Chromosomen zur Cytologie von Escherichia coli. Archives of Microbiology, 40, 283.

10. Scherbaum, O., \& Rasch, G. (1957). Cell size distribution and single cell growth in Tetrahymena. Pyriformis GL, 41(3), 161182.

11. McMillen, D., Kopell, N., Hasty, J., \& Collins, J. J. (2002). Synchronizing genetic relaxation oscillators by intercell signalling. Proceedings of the National Academy of Sciences of the United States of America 99, 679.

12. Pelling, A. E., Sehati, S. B., Gralla, E. B., Valentie, J. S., \& Gimzewski, J. K. (2004). Local nanomechanical motion of the cell wall of Saccharomyces cerevisae. Science, 305, 1147. 
13. Ko, K. S., Arora, P. D., \& McCulloch, C. A. G. (2001). Cadherins mediate intercellular mechanical signaling in fibroplats by activation of stretch-sensitive caclium-permeable channels. Journal of Biological Chemistry, 276, 35967.

14. Kubitschek, H. E. (1969). Growth during the bacterial cell cycle analysis of cell size distribution. Biophysical Journal, 9, 792

15. Haase, R. (1963). Thermodynamik der Irreversiblen Prozesse (p. 119). Darmstadt: Steinkopf.

16. Haase, R. (1956). Thermodynamik der Mischphasen (p. 274). Berlin: Springer.

17. Micoulet, A., Spatz, J. P., \& Ott, A. (2005). Mechanical response analysis and power generation by single-cell stretching. Chemical Physics Chemistry, 6, 663-670.

18. Koepf, M., \& Kilian, H. G. (1999). Relaxation in the glass transition regime interpreted in terms of the aggregate. Model Acta Polymer, 50, 109.

19. Uhlig, G. (1979). Entwicklung beim Seeigel (Psamechninus miliaris) I. Befruchtung und Furchung, Film C 1187 IWF, Sektion Biologie, Serie 12, Nr. 23.

20. Treloar, L. R. G. (1975). The physics of rubber elasticity (3rd edn., p. 64). Oxford: Clarendon Press.

21. Kilian, H. G., Zink, B., \& Metzler, R. (1997). Aggregate model of liquids. Journal of Chemical Physics, 107, 8697.

22. McCrum, N. G., Read, B. E., \& Williams, G. (1967). Anelastic and dielectric effects in polymer solids (p. 102). London, New York, Sydney: Wiley.

23. Bartkowiak, D., Nothdurft, W., \& Röttinger, E. M. (1999). Differential proliferation dependence of $\alpha$ and $\beta$ damage in
$\mathrm{X}$-irradiated Chinese Hamster cells. Strahlentherapie Onkologie, $175,239$.

24. Schwittay, C., Mours, M., \& Winter, H. H. (1995). Rheologiocal expression of physical gelation in polymers. Faraday Discussions, 101, 93.

25. Kohlrausch, F. (1968). Praktische Physik (vol. 3, pp. 28-29). Stuttgart: Teubner BG.

26. Schaechter, M., Maaloe, O., \& Kjeldgaard, N. (1958). Dependency on medium and temperature of cell size and chemical composition during balanced growth of Salmonella tryphimurium. Journal of General Microbiology, 19, 92.

27. Alberts, B., Bray, D., Johnson, A., Lewis, J., Raff, Roberts, K., \& Walter, P. (1997). Essential cell biology (p. 571). New York, London: Garland.

28. Kemkemer, R. (2002). Zellform und Zellorientierung von Melanozyten-Physikalische Untersuchungen zu zellulären Maschinen, Thesis Naturwiss. Fakultät University of Ulm.

29. Schneider, B. M. (1984). Untersuchungen über den Mercaptoenthanaol stimulierbaren Faktor (Ma3F) im menschlichen Serum. Thesis, Fakultät für Klinische Medizin Universität Ulm.

30. Czihak, G., Langer, H., \& Ziegler, H. (1981). Biologie (3rd edn., p. 737). Heidelberg, New York: Springer.

31. Taylor, C. R., \& Weibel, E. R. (1981). Design of the mammalian respiratory system. I. Problem and strategy. Respiration Physiology, 44, 1-10. 